La corsa, il puledro e il frustino

La storiografia contemporaneistica italiana periodicamente si analizza, o viene sottoposta a esame. Lo fu al lontano convegno di Perugia del 1967, quando da pochi anni essa aveva conosciuto i primissimi concorsi universitari, appunto, per cattedre di Storia contemporanea. L'associazione degli storici propose un altro resoconto, un paio di decenni più tardi. Ma non si trattava di bilanci incontestati, anche per via della pluralità di anime dentro la stessa disciplina. Furono più utili e più icastici gli strali di Ruggiero Romano, di certi saggi paludati. Più di recente, produttivi sono stati anche certi sguardi esterni.

La ricerca storico-contemporaneista - come ogni disciplina - interroga se stessa in occasioni rituali, come per esempio al momento della pubblicazioni di grandi opere, meglio se collettive, o alla scomparsa di grandi figure, o in alcune ricorrenze particolari. Dal 1990 è attiva la Società italiana per lo studio della storia contemporanea (Sissco), e anche ad essa si devono alcuni bilanci. Nel redigere un bilancio produttivo, però, la rilevanza dell'equilibrio non deve far perdere di vista l'importanza di un punto di vista, ché altrimenti il resoconto si fa asettico, e sterile, ed anche noioso. Perché i bilanci che non fanno discutere o sono perfetti o incidono poco.

Quando abbiamo letto la pungente rassegna di Christof Dipper, pubblicata come Die italienische Zeitgeschichtsforschung. Eine Momentaufnahme, dai "Vierteljahrshefte für Zeitgeschichte" (vol. 63 n. 3, del luglio 2015, pp. 351378), abbiamo pensato che un dibattito si sarebbe sviluppato in Italia. Noto era il suo autore, prestigiosa la sede di pubblicazione, significativo era il suo testo, che si segnalava per un singolare impasto di verità - in Italia spesso sussurrate a mezza voce ma mai pubblicamente ammesse -, qualche descrizione affrettata, qualche stereotipo: utili per semplificare in tutto sommato poche pagine in breve a un pubblico non italiano le complessità della ricerca storica di un altro Paese, anche quando non sostenibili. Inoltre, qualche pennellata veloce è comprensibile quando non si mira a una esaustiva rassegna storiografica su un tema specifico ma si dipinge un panorama generale.

Il dibattito che ci attendevamo però non c'è stato. Il silenzio ha prevalso. $E$ mese dopo mese, la situazione ci è parsa sempre più imbarazzante. E imbaraz- 
zato. E grave. Tutto ciò nonostante Dipper - che aveva fatto parte della prima commissione per l'Abilitazione scientifica nazionale (Asn) - basasse il suo ragionamento su molti dati di fatto.

Di suo, "Italia contemporanea" ha fatto tradurre l'articolo e l'ha fatto leggere ad alcuni diversi studiosi. Uno storico assai apprezzato, appena uscito dai ruoli, componente del Gev del recente esercizio di Vqr. Il presidente della Sissco in carica. Una storica da tempo sensibile all'innovazione storiografica $e$ alle tematiche di genere. Due giovani studiosi, che avevano già in precedenza analizzato (e criticato) quella università e quella disciplina da cui (sino a oggi) erano ancora fuori. Ne è nato un dibattito con posizioni diverse, che ora ringrazia lo studioso tedesco per la chiaroveggenza che il distacco gli permette e ora ne prende le distanze per certe sue rapidità giudicate eccessive; un dibattito con posizioni diverse fra giovani e meno giovani, fra "strutturati" e chi invece è (ancora?) di là dal "crinale del fuori ruolo".

$\mathrm{Ne}$ è uscito un dibattito non ideologico, fra "scuole" o "correnti" o "famiglie". Certo, si legge sotto certe frasi il richiamo anche a questo. In ogni caso il dibattito e la differenza di posizioni sono ora sotto gli occhi dei lettori. E sono più chiari, sia pur nella brevità degli interventi, di tante allocuzioni a convegni associativi o rituali. Discutere non è un male, per una disciplina scientifica.

Peraltro, ci pare, ragioni per il dibattito ci sarebbero. Un'occasione avrebbe potuto essere proprio il concluso cinquantennio di vita (stando ai concorsi appunto per Storia contemporanea e non più solo per Storia del risorgimento): ma non è stato colto. Forse non è stata celebrata perché c'è poco da festeggiare: in questi stessi anni il numero di docenti riconosciuti accademicamente è fortemente diminuito, il pensionamento di un'intera generazione ha assai indebolito la disciplina, $i$ libri di storia contemporanea non si vendono più nel numero di copie di una volta, le riviste (che, curiosamente, si moltiplicano) vendono sempre meno, gli studiosi che "fanno" la ricerca storico-contemponeistica sono ancora divisi non solo in diversi "settori scientifico-disciplinari" ma addirittura in "aree" diverse, le biblioteche universitarie italiane sono in enorme difficoltà ad aggiornarsi sulle tante innovazioni della ricerca internazionale, con la conseguenza che la necessaria internazionalizzazione degli studi storici nazionali non è certo aiutata, mentre anche le fondazioni di studi storici non brillano per fondi, e non ne sono nate di nuove, mentre le forme aggregative della disciplina hanno conosciuto una brusca riarticolazione (da un intreccio di appartenenze ideali e fedeltà locali è passata ad un miscuglio di localismo e chissà cosa altro), mentre le forme della sua riproduzione - a partire dai dottorati di ricerca - sono indebolite da una diminuzione generale delle risorse, applicate ma con logiche random.

A bilanciare le difficoltà ci sono, ovviamente, anche i successi. Una parte della produzione storiografica dei contemporaneisti e delle contemporaneiste d'Italia è sicuramente di qualità eccezionale. I loro lavori sono pubblicati anche in altre lingue ed entrano nei dibattiti internazionali. Da altri Paesi si vie- 
ne in Italia non solo per studiare la storia d'Italia ma anche per vedere come studiano gli storici e le storiche della Penisola, quali metodi elaborano, come interpretano le fonti. Alcune riviste di storia contemporanea italiana sono diffuse in biblioteche non italiane e (per quanto talora con criteri incomprensibili) alcune stanno anche nei grandi database aggregatori privati internazionali. I successi non mancano, per quanto la loro elencazione si impone qui più breve rispetto all'indicazione delle difficoltà per un tratto di modestia.

Proprio perché $i$ successi ci sono, ignorare che ci siano "cose che non vanno" o essere internazionali a intermittenza facendo finta di non aver letto contributi critici di apprezzati studiosi stranieri sono comportamenti miopi che non fanno onore alla disciplina. Riviste, associazioni, fondazioni, "scuole", "famiglie" non possono ignorare le critiche, a meno di non voler apparire poco scientifiche, e non aiutano il futuro.

La corsa è molto difficile, ma ignorare il frustino - che lo si condivida in tutto o in parte - vantando il puledro non aiuterà a farlo vincere. Per chi la volesse utilizzare, "Italia contemporanea" è e rimane una sede critica ed aperta al dibattito. 


\title{
La storia contemporanea in Italia vista dalla Germania Un'istantanea
}

\author{
Christof Dipper*
}

Le prospettive sono fosche, insider critici parlano addirittura di un "suicidio" delle università italiane, riferendosi con questo termine anche alla storiografia contemporanea a sud delle Alpi. Christof Dipper, un veterano particolarmente stimato della ricerca storica sull'Italia, ha avuto la possibilità di formarsi una propria opinione sulla situazione della storiografia contemporanea italiana, in qualità di membro di una commissione valutativa. Come si evince dalla sua lucida analisi, originariamente pubblicata come Die italienische Zeitgeschichtsforschung. Eine Momentaufnahme, in "Vierteljahrshefte für Zeitgeschichte", a. 2015, n. 3, pp. 351-378, c'è poco spazio per l'ottimismo: ovunque si volga lo sguardo si incappa in autoreferenzialità, provincialismo e tradizionalismo metodologico, mentre coraggio e forza innovativa sono riscontrabili solo in poche mosche bianche.

Parole chiave: Storiografia, Storia contemporanea, Italia, Abilitazione scientifica nazionale (Asn)

\section{Contemporary History in Italy: a View from Germany}

The perspectives are grim. Insiders have been talking about the risk that Italian universities might be committing suicide, along with Italian contemporary historiography. As a member of Miur's evaluation committee, Christof Dipper, a well-known historian of Italy, recently had the chance reflect on the condition of the country's contemporary historiography. As Dipper clearly points out, there is little room for optimism, since most publications are self-referential, parochial in content and very parochial from the point of view of their methodology. Only a few exceptional studies are innovative and ground-breaking.

Key words: Historiography, Contemporary History, Research Assessment (Asn)

\section{Le università nell'orbita della politica}

Le università in Italia, nel Paese che ha dato loro origine, si trovano in una situazione delicata. Sostanzialmente tale affermazione vale in generale per tutte le istituzioni della ricerca. La causa diretta è ovviamente la mancanza di denaro. Il paese spende al momento, con una tendenza decrescente, poco più dell'1 per cento del Pil per la ricerca ${ }^{1}$, contro il 3 per cento attuale della Repubblica

* Technische Universität Darmstadt; dipper@pq.tu.darmstadt.de

${ }^{1}$ Marco Meriggi riporta 1,25 per cento per la ricerca scientifica in Italia e 2,92 per cento in Germania. Si veda Zur Lage der Nachwuchsforschung in der italienischen Geschichtswissen- 
federale tedesca. La mancanza di fondi non è un fenomeno nuovo. Com'è stato recentemente sottolineato, fin dal 1861 con poche eccezioni istruzione e ricerca sono sempre state marginali per le élite politiche. Anche quando i politici del regno d'Italia definivano la "scienza nazionale" un obiettivo centrale, si trattava in realtà di parole vuote ${ }^{2}$. Proprio Mussolini sostenne per la prima volta in maniera massiccia le scienze naturali, ma quando il loro finanziamento divenne troppo costoso diede priorità agli armamenti ${ }^{3}$. Dalla fine degli anni Trenta le scienze naturali furono ricollocate laddove le altre discipline erano sempre rimaste: fra le "questioni secondarie".

Ciò rimanda alla causa profonda: la mancanza di volontà da parte delle élite politiche di impegnarsi seriamente per l'istruzione e la scienza e in favore delle loro strutture organizzative. Il problema dunque non sta nella mancanza di fondi. L'Italia dopotutto non è un paese in via di sviluppo. Il denaro è utilizzato per altre cose. È stato Mario Monti, autorevole professore di economia, in qualità di presidente del Consiglio ad aver sacrificato una parte dei fondi destinati alla scienza e alla ricerca per il salvataggio di Alitalia. Durante il suo mandato - o a causa di questo? - è stato interrotto il processo di finanziamento alla ricerca di base, mediante i bandi Prin. Va da sé che, prima ancora, Berlusconi non fosse disposto a dare denaro alle università, considerati i suoi obiettivi politici e la rosa di valori dei suoi sostenitori ${ }^{4}$.

schaft. Ein Gespräch mit Marco Meriggi und Elena Mazzini, "Weltweit vor Ort. Das Magazin der Max-Weber-Stiftung", 2014, n. 2, pp. 23-26, qui p. 26. Alle università sono andati nel bilancio per il 2014 sette miliardi di euro (pari allo 0,43 per cento del Pil), ridotti ulteriormente nel 2015 fino a un massimo di 400 milioni di euro, taglio voluto dal governo Renzi per favorire l'assunzione di 148.000 insegnanti in possesso fino ad allora soltanto di un contratto a tempo determinato. Questo è il punto di arrivo di una "decennale eutanasia" del sistema universitario italiano; si veda Novello Morelli, Come ti rottamo l'università, "Mente politica" del 18 ottobre 2014, http://www.mentepolitica.it/articolo/come-ti-rottamo-la-universit/237 (ultimo accesso 8 luglio 2016), p. 1. Per un confronto: il ministero della scienza, ricerca e arte del Land di Baviera ha messo a bilancio, nel piano preventivo per il 2014, 6,1 miliardi di euro, http://www.stmf.bayern. de/haushalt/staatshaushalt_2013/haushaltsplan/, piano specifico n. 15, p. 1180 (ultimo accesso 8 luglio 2016); le cifre ad ogni modo non sono direttamente confrontabili tra loro.

${ }^{2}$ Si veda Anna Gianna Manca, Struttura ed evoluzione dell'amministrazione della pubblica istruzione nella monarchia costituzionale italiana (1861-1922), in Annali/Jahrbuch des Italienisch-Deutschen Historischen Instituts in Trient, (2010/11), n. 36/37, pp. 175-215. Il fallito orientamento al modello della ricerca tedesco è descritto in modo eccellente da Francesco Marin, Die "deutsche Minerva" in Italien. Die Rezeption eines Universitäts- und Wissenschaftsmodells 1861-1923, Köln 2010.

${ }^{3}$ Sull'argomento si veda Christof Dipper, Nationalsozialistische und faschistische Wissenschaftspolitik im Vergleich, in Klaus Kempf e Sven Kuttner (hrsg.), Das deutsche und italienische Bibliothekswesen im Nationalsozialismus und Faschismus. Versuch einer vergleichenden Bilanz, Wiesbaden, Harrassowitz Verlag, 2013, pp. 1-36.

${ }^{4}$ Il secondo governo Berlusconi stabilì per gli anni 2009-2013 tagli al personale nelle università per un ammontare di 1,4 miliardi di euro; questa somma fu successivamente ridotta a 0,9 miliardi. La ricerca tecnica di eccellenza ottenne fondi straordinari. Si veda Giulio Palermo, L'università dei baroni. Centocinquant'anni di storia tra cooptazione, contestazione e mercificazione, Milano, Punto rosso, 2011, p. 119. Le discutibili affermazioni politiche di questo libro non saranno di seguito né riportate né tanto meno utilizzate. 
Tutto ciò ha ovviamente delle conseguenze. Una di queste è la fuga di massa delle persone istruite all'estero. Nel 2012 il 25 per cento degli espatriati aveva una laurea o un titolo di studio di livello più alto ${ }^{5}$. La meta primaria però non è la Germania: nel quinquennio 2005-2010 tra gli immigrati in quel paese con titolo accademico gli italiani occupano, fra tutti i paesi dell'Europa meridionale, la penultima posizione ${ }^{6}$. Al contrario gli italiani sono fortemente rappresentati nelle statistiche dell'European Research Council di Bruxelles. Solo i tedeschi lasciano in proporzioni ancora maggiori il proprio paese, con la differenza che altrettante persone interessate alla ricerca si orientano verso la Germania; un ricercatore straniero invece difficilmente sceglie l'Italia ${ }^{7}$. O forse, vorrebbe anche, ma non può, perché il Paese non ha approntato allo scopo sufficienti infrastrutture. Solo per citare il caso delle scienze umane, mancano del tutto istituti di ricerca degni di questo nome, posti per visiting professors, programmi per borse di studio, rappresentanze all'estero. Ed è ovviamente comprensibile che quando già scarseggiano i mezzi per i propri ricercatori non vi sia alcuna risorsa per gli stranieri.

A un primo sguardo si ha l'impressione che le differenze fra Germania e Italia - per ovvie ragioni la comparazione si limita a questi due paesi - non siano poi così grandi e che in realtà nel confronto l'Italia ottenga a prima vista dei risultati per nulla negativi. Nel 2005 c'erano nelle università italiane 2.078 ricercatori e ricercatrici delle scienze storiche, in quelle tedesche solo $1.625^{8}$. Vi-

${ }^{5}$ Si veda Marco Mondini, Qualità vo cercando. De-meritocrazia e ope legis uccidono la scienza, impoveriscono il paese (e annientano una generazione), "Mente politica" del 3 giugno 2014, http://www.mentepolitica.it/articolo/de-meritocrazia-e-ope-legis-uccidono-la-scienza/75 (ultimo accesso 8 luglio 2016); nel 2009 i laureati con titolo di studio universitario antecedente al processo di Bologna rappresentavano circa il 15 per cento della popolazione italiana. Si veda Daniela Del Boca e Anna Giraldo, Why Has the Growth of Female Employment in Italy Been so Slow?, "Journal of Modern Italian Studies", 2014, n. 18, pp. 485-499, Tab. 1.

${ }^{6}$ Berlin-Institut für Bevölkerung und Entwicklung, Neue Potenziale. Zur Lage der Integration in Deutschland, Berlin 2014, p. 3 (disponibile in formato pdf in: http://www.berlin-institut. org/publikationen.html) (ultimo accesso 8 luglio 2016).

${ }^{7}$ Cifre e grafici in European Research Council, Annual Report on the ERC activities and achievements in 2013, Bruxelles 2014 (consultabile all'indirizzo: http://erc.europa.eu/publications) (ultimo accesso 8 luglio 2016), nonché ERC in a nutshell, update 17/03/2014, http://erc.europa.eu/press_release/erc-nutshell (ultimo accesso 8 luglio 2016).

${ }^{8} \mathrm{Si}$ veda Ilaria Porciani, Lutz Raphael (eds.), Atlas of European Historiography. The Making of a Profession 1800-2005, Basingstoke, Palgrave Macmillan, 2010, p. 121 e p. 164. Vi è inoltre in Germania un gran numero di storici attivi in istituti di ricerca al di fuori del mondo universitario, mentre in Italia qualcosa del genere quasi non esiste. I numerosi istituti storici della Resistenza per esempio, al pari di altri istituti di storia contemporanea per la ricerca e la memoria, con proprie collane di pubblicazioni, sono quasi esclusivamente gestiti da docenti delle scuole secondarie distaccati, o comandati. Tra le poche eccezioni vi è l'Istituto storico italo-germanico (Isig) di Trento, fondato nel 1973, gestito dalla provincia autonoma di Trento e l'istituto di ricerca (non universitario) Centro di documentazione ebraica contemporanea (Cdec), con sede a Milano, una fondazione Onlus che riceve circa un terzo dei suoi fondi dallo Stato. Secondo Stefano Cavazza, Die Neueste Geschichte in Italien. Überlegungen zu einem etablierten Fach, "Anna- 
ste in quest'ottica, le disfunzioni a sud delle Alpi avrebbero dovute essere cinquant'anni fa, quando il rapporto era di 93 a 451, ben più gravi di oggi, almeno sul piano aritmetico. Allora però non solo l'accesso agli studi universitari era in Italia particolarmente ristretto e un percorso professionale dello storico ancora sconosciuto — è stato introdotto solo nel 1978 —, ma c'erano allora ancora una burocrazia ministeriale con chiare funzioni statocentriche di comando e uno spirito di corpo accademico che non ostacolava i criteri della ricerca scientifica. Insieme questi due elementi facevano sì che sui ricercatori gravassero grandi aspettative e che il cursus honorum seguisse regole severe. Ciò significava che lo studioso solo dopo un vaglio accurato veniva promosso inizialmente come professore, di norma (come anche in Germania) con un titolo di assistente o di libero docente, nel Sud o nelle isole, dove lo si osservava, prima di lasciarlo tornare per un impiego in una delle (poche) prestigiose università dell'Italia centrale e settentrionale?.

Tutto ciò è ormai storia. Come in Germania, anzi come in quasi tutta Europa, anche in Italia a partire dagli anni Settanta la legislazione sull'università è diventata oggetto dei giochi della politica ed è quasi permanentemente soggetta a cambiamenti. Si tratta spesso di esperimenti inevitabili e comunque riscontrabili anche altrove, volti a canalizzare l'impetuosa crescita dinamica del settore dell'istruzione iniziata negli anni Sessanta. In Italia questa avvenne in realtà in forme più modeste che altrove: tra il 1950 (in particolare dall'apertura degli studi universitari a tutti i diplomati nel 1969) e il 1995 il numero degli studenti si è sestuplicato, mentre in Germania il numero è aumentato di nove volte. La risposta della politica, detto in soldoni, stava nella pluridecennale apertura dei canali del personale universitario, cosa che non beneficiava alla sua qualità e imponeva allo Stato ulteriori sforzi finanziari. Retrospettivamente emerge l'eliminazione in molti passaggi dei criteri di efficienza delle università validi anche a livello internazionale e il conseguente "suicidio" commesso dalle università italiane ${ }^{10}$. Già nel primo ventennio dopo il 1960 il corpo docenti si è sestuplicato ${ }^{11}$ : nel 1967 furono create 1000 cattedre e altre 7.500 nel 1973 . $\mathrm{Fu}$ inoltre disposta la nomina a professore straordinario di migliaia di persone

li/Jahrbuch des Italienisch-Deutschen Historischen Instituts in Trient”, 2010/11, n. 36/37, pp. 82111, qui p. 86, nota 8, vi sarebbero per l'anno 2011477 storici contemporaneisti "puri" di tutte le categorie, però senza alcuna disciplina affine, come per esempio nordamericanistica ecc.

${ }^{9}$ Alcuni esempi: Federico Chabod iniziò nel 1934 a Perugia, dove era stata appena fondata la facoltà di Scienze politiche, andò poi nel 1938 a Milano e nel 1946 a Roma. Delio Cantimori nel 1939 iniziò a Messina e fu chiamato già nel 1940, grazie alle sue protezioni politiche, a Roma. Franco Venturi prese il suo primo incarico a Cagliari nel 1951, prima di andare a Genova e poi a Torino nel 1958. Infine Renzo De Felice iniziò a Salerno nel 1968, prima di trasferirsi a Roma nel 1972.

${ }^{10}$ L'immagine drastica è utilizzata da Mondini, Qualità vo cercando, cit., che descrive anche la maggior parte degli eventi riportati di seguito.

${ }^{11}$ Tutte le cifre in Guy Neave, Patterns, in Walter Rüegg (ed.), History of the University in Europe, vol. 4, Cambridge University Press, Cambridge 2011, pp. 31-69, qui p. 41. 
che avevano raggiunto in qualche modo ruoli d'insegnamento ${ }^{12}$. Ma non finisce qui. Con un decreto del presidente della Repubblica del 1980 negli anni successivi il restante personale universitario, si parla di decine di migliaia di assistenti e borsisti, passò nelle nuove posizioni a tempo indeterminato di ricercatori e professori associati ${ }^{13}$. La riforma universitaria, di cui era responsabile Salvatore Valitutti ${ }^{14}$, si è lasciata alle spalle seri danni e, considerata l'aspettativa di vita degli allora beneficiari dei provvedimenti, difficili da riparare in un prossimo futuro.

Conseguentemente la cosiddetta riforma Ruberti del 1989/90 riguardava questioni completamente diverse. Il suo artefice, un informatico profondo conoscitore delle tendenze internazionali ${ }^{15}$, cercò di importare anche in Italia l'allora tipica trasformazione della gestione universitaria in direzione della responsabilizzazione e dell'autonomia ${ }^{16}$. In termini di personale non cambiò molto. Solo i ricercatori furono valorizzati come membri paritari nel corpo docente, mentre avevano già sempre avuto gli stessi obblighi d'insegnamento dei professori. Ciò faceva risparmiare soldi allo Stato ma danneggiava ovviamente la ricerca, per la quale questa forma di impiegati era stata originariamente (e presumibilmente) assunta. Ancor più rilevante fu che Ruberti favorì alle università, quindi ai suoi colleghi, un'ampia autonomia, che includeva gli indirizzi di studio, gli statuti, l'utilizzo dei fondi ecc. - una totale rottura con la tradizione, che divenne ancora più evidente nel momento in cui Ruberti permise alle università di accettare risorse di terzi. La sinistra vi intravide la consegna delle università al capitale e ai suoi interessi - a confutare queste bizzarre idee basterebbe l'esempio di fondazioni che sostengono gli studiosi delle scienze umane e sociali, come fanno la fondazione Henkel e la Thyssen, ma che in Italia non esistono - e protestò di conseguenza, ovviamente per nulla. Dopo le dimissioni di Ruberti da ministro, un'ulteriore serie di leggi ampliò l'autonomia finanziaria in favore dei settori scientifico-disciplinari già esistenti sul modello france-

${ }^{12}$ Si veda Palermo, L'università dei baroni, cit., p. 57.

${ }^{13}$ In Germania un fenomeno simile è noto in Assia, dove negli anni Settanta fu cooptata una parte considerevole dei ricercatori privi di abilitazione al posto di professore associato. La legge si attirò dure critiche e fu irrisa dai commenti degli accademici ("Discount-Professor"). L'esperimento fu rapidamente interrotto. Una nomina in altri Länder, in particolare in quelli guidati dalla Cdu, era sostanzialmente esclusa.

${ }^{14}$ Salvatore Valitutti è stato a lungo alto funzionario del ministero dell'istruzione, poi deputato del partito liberale e chiuse la sua carriera come ministro nel 1979/80. Il nuovo ordinamento del sistema universitario, da lui avviato, fu realizzato dopo il suo ritiro.

${ }^{15}$ Antonio Ruberti, professore d'informatica e rettore della Sapienza di Roma, è stato ministro senza portafoglio nel ministero dell'Istruzione tra il 1987 e il 1989, poi fino al 1992 ministro dell'Università e della Ricerca, per passare in seguito a Bruxelles come commissario Eu per la ricerca.

${ }^{16}$ Per un quadro internazionale si veda Geoffrey Lockwood, Management and Ressources, in Rüegg (ed.), History of the University, cit., pp. 121-152, qui pp. 137 sg. 
$\mathrm{se}^{17}$, che concepiva le discipline in maniera parzialmente nuova e che portò al riordino di un gran numero di cattedre. Ciò rese discutibile agli occhi di molti candidati la procedura di assunzione compiuta per questa via; i ricorsi si susseguirono con una tale frequenza da costringere i tribunali amministrativi ad annullare interi concorsi. L'effetto a valanga allarmò la politica, che riordinò completamente le procedure con la cosiddetta riforma Berlinguer.

Per Luigi Berlinguer ${ }^{18}$ erano prioritari lo snellimento delle procedure di assegnazione dei posti e la riduzione dei ricorsi; a tal fine la legge del 3 luglio 1998 tolse la gestione delle assunzioni al centro romano, cioè al ministero dell'università. Nel momento in cui il ministero consegnava totalmente la formulazione dei bandi e le assunzioni alle università, ci si trovò di fronte a un'ulteriore rottura con 130 anni di tradizione. Inoltre la legge circoscrisse la richiesta con l'introduzione di una tassa sulle domande di partecipazione ai concorsi e stabilì un tetto massimo di cinque domande all'anno per candidato. Nuova era anche la distinzione che si faceva, come nei paesi di lingua tedesca, tra esame attitudinale e nomina, oltre al fatto che il primo degli sconfitti ai concorsi, previo secondo posto pari passu in graduatoria, potessero ottenere la nomina da qualche altra parte senza altre procedure. Questa clausola tuttavia, seppur pensata in buona fede, si è rivelata in realtà una corsia preferenziale per protezioni e accordi reciproci. Sia Berlinguer, sia il suo presidente del consiglio Romano Prodi, sebbene entrambi insegnassero nelle università, non hanno evidentemente intuito i danni potenziali di un'autonomia delle università liberate da controlli nel paese del familismo e del clientelismo. A ogni modo i cosiddetti concorsi Berlinguer si sono di fatto trasformati in promozioni di massa e il numero dei professori ordinari è aumentato da 13.000 a 19.000 . Questo processo spiega anche il già citato maggior numero di storici in Italia rispetto alla Germania.

Nel periodo inizialmente dominato da Forza Italia e, a partire dal 2011, dagli avversari politici di Berlusconi, le politiche universitarie sono consistite nella contraddittoria combinazione di tentativi volti, da un lato, a garantire la qualità e, dall'altro, a ridurre le risorse. Come ministro del governo Berlusconi Letizia Moratti ${ }^{19}$ si è fatta particolarmente odiare dal personale universitario, in particolare per la reintroduzione dei concorsi centralizzati, per la valo-

${ }^{17}$ Parigi aveva ad ogni modo creato già nel 1968 per le sue università l'Unités d'enseignement et de recherche.

${ }^{18}$ Luigi Berlinguer, storico del diritto e membro del Pci fino al suo scioglimento, è stato fra il 1996 e il 2000 ministro per l'Università e la Ricerca e prima di allora rettore dell'università di Siena. Tra il 2010 e il 2014 è stato parlamentare europeo per il Partito democratico e da allora riveste il ruolo di consulente del ministero dell'Istruzione.

${ }^{19}$ Dirigente milanese, stretta collaboratrice di Berlusconi, in seguito sindaco della sua città, dove le sue politiche del personale simili al modello americano dello spoil-system (chiamando non meno di 54 persone esterne nell'amministrazione) sono state dichiarate "censurabili sotto diversi profili" da un tribunale. Ricandidata, ha perso le elezioni, nonostante avesse portato l'Expo a Milano per il 2015. 
rizzazione delle università private e per corrispondenza e per diverse misure con cui le università sono state spinte a cooperare con l'economia (locale). È rimasta la possibilità, già introdotta dal suo predecessore Berlinguer, di assumere direttamente studiosi affermati, tuttavia la normativa di recepimento - fino al 10 per cento dei posti liberi di associati e ordinari poteva essere occupato in tal modo - rivela come si sia poi pensato in misura minore alle eccellenze. Il successore della Moratti scelto dalla sinistra rimase in carica solo 12 mesi senza lasciare tracce, mentre il successivo governo, nuovamente formato da Berlusconi, fu dominato dalle politiche sulla ricerca influenzate dal ministro delle Finanze Giulio Tremonti, il quale si rese celebre per la più ampia riduzione fiscale dai tempi della Seconda guerra mondiale. La politica scientifica ricevette perciò un'impronta del tutto singolare e disomogenea. Da un lato la ministra Mariastella Gelmini ${ }^{20}$, in aperta violazione della costituzione, ha ridistribuito il bilancio delle università a favore delle ormai numerose università private e ha fortemente ostacolato l'erogazione di risorse di bilancio alle università, apparentemente motivando il provvedimento con la lotta alla mafia (la quale fino ad allora non sembrava interessarsi alle università). Dall'altro ha cercato di riportare un principio concorrenziale nelle università. Entrambe le misure hanno suscitato vive proteste; nel 2008 vi furono le più grandi manifestazioni da alcuni decenni a questa parte, che videro uniti studenti della scuola e dell'università con il personale accademico. Dall'ottica tedesca una delle principali riforme della ministra risulta familiare: i nuovi ricercatori a partire dalla riforma Gelmini del 2010 ricevono solo contratti a termine (3 anni più 3), il tutto nell'ambito di piani del personale drasticamente ridimensionati, cosicché la maggior parte di essi non ha alcuna prospettiva di pervenire a un impiego fisso, men che meno a un posto di lavoro a tempo indeterminato. La logica conseguenza è stata un precariato accademico che fino ad allora era noto solo in Germania, lì però come risultato principalmente del successo della ricerca finanziata da terzi ${ }^{21}$.

Nella riforma Gelmini rientra anche la decisione di valorizzare l'abilitazione introdotta da Berlinguer, trasformandola nell'Abilitazione nazionale scientifica, uno strumento volto al controllo centrale degli standard minimi della ricerca scientifica. Con essa l'idoneità non è più stabilita dalle università locali, ma è conseguente a una procedura severa che - in sede della sua prima applicazione - ha coinvolto studiosi anche provenienti dall'estero.

${ }^{20}$ Mariastella Gelmini, avvocato, è stata tra il 2008 e il 2011 ministro dell'Istruzione, dell'Università e della Ricerca nel quarto gabinetto Berlusconi.

${ }^{21}$ In Germania nel 2009 circa l' 85 per cento del personale scientifico nelle università era impiegato in posizioni di ricercatore, la maggior parte in incarichi di qualificazione e/o finanziati da terzi. Solo una piccola parte del personale di ricerca, in crescente contrazione, ha un impiego a tempo indeterminato. Il numero dei posti di professore ordinario si attesta sul 13 per cento — una quota così ristretta è limitata all'ambito dei paesi di lingua tedesca e dell'Europa centroorientale; le cifre si trovano in Reinhard Kreckel, Habilitation versus Tenure Track. Kurzfassung, in Aus Forschung und Lehre, 2012 (http://www.academics.de/wissenschaft/habilitation versus_tenure_track_51642.html) (ultimo accesso 18 luglio 2016). 
Prima di valutare i risultati di questa procedura, si tenterà di tratteggiare la cultura universitaria italiana vista dalla prospettiva tedesca $(\mathrm{Cd} 1)$ mediante cinque parole chiave.

Primo: il centralismo. La tradizionalmente diffusa sfiducia della burocrazia romana verso gli organi locali vale anche per le università, le quali sono conseguentemente tenute a una briglia molto corta e per ogni singola decisione devono procurarsi l'autorizzazione da Roma ${ }^{22}$. Ciò impone lo stabilirsi di reti di rapporti per sopravvivere nel quotidiano. Dalla prospettiva tedesca il divieto (temporaneo) per i rappresentanti locali della materia alla distribuzione dei posti da professore è altrettanto incomprensibile quanto quello di aprire al finanziamento dei privati. Anche l'impossibilità di conseguire l'abilitazione nella Facoltà di riferimento appare a un primo sguardo singolare, ha tuttavia un senso nel momento in cui è permessa la nomina interna.

Secondo: i ricercatori. Questi sono scelti con bandi nazionali, ma per i professori ci sono ovviamente sotterfugi per sistemare i propri allievi. Che la legge del 1980 non riguardasse né la ricerca né il reclutamento, bensì il beneficio, si evince dal fatto che i ricercatori fino al 2010 sono stati assunti a tempo indeterminato, con la conseguenza che al momento una parte considerevole dei ricercatori è costituita da sessantenni senza ulteriori titoli oltre la laurea ma, in compenso, con lo stesso carico d'insegnamento dei professori ordinari. Naturalmente ciò non esclude risultati eccellenti nei singoli casi.

Terzo: la rappresentanza degli interessi. Negli impiegati statali i sindacati italiani trovano il loro ultimo appiglio. Qui la regola è costituita da una rappresentanza degli interessi senza alcun riguardo nei confronti della collettività dei contribuenti e con un'enfasi sempre orientata alla lotta di classe. Ulteriori difficoltà sono conseguenza del fatto che un gran numero di sindacati si fanno concorrenza e cercano quindi di proporre un linguaggio sempre più radicale. Inoltre ogni categoria professionale, fino ai collaboratori, ha una sua rappresentanza nazionale, e così anche i professori. Una mentalità assistenziale estranea all'ambito scientifico e il rifiuto del principio concorrenziale in ampi settori del personale accademico si spiegano con questa situazione riscontrabile in molti Paesi latini.

Quarto: i professori. Questi sono politicamente divisi. Avevano tuttavia in passato un potere molto più ampio rispetto agli ultimi vent'anni e godono o risentono - dei vantaggi di un sistema d'insegnamento da sempre scolarizzato. Offrono non sempre seminari ed esercitazioni, per ogni singolo studente

${ }^{22}$ La burocrazia italiana è tanto imponente quanto inefficiente. Sull'argomento si guardi la recente ricerca, impostata particolarmente bene da Magda Bianco e Giulio Napolitano, Why the Italian Administrative System is a Source of Competitive Disadvantage, in Gianni Toniolo (eds.), The Oxford Handbook of the Italian Economy since Unification, Oxford, Oxford Handbooks, 2013, pp. 533-568. Francesco Paolo Cerase, "Performance" ed efficienza del settore pubblico italiano: comparazione con Francia, Germania, Giappone, Regno Unito (1980-2010), "Amministrare 45", 2015, n. 1, pp. 33-73 calcola nell'ambito di una comparazione internazionale un peggioramento fin dal 1980. 
da correggere c'era spesso solo una volta la tesi di laurea, a partire dal processo di Bologna due volte. Le infrastrutture per il loro lavoro sono insufficienti: si divide con i colleghi uffici, telefono, fax, matite e carta; di conseguenza raramente si incrociano i colleghi all'università. L'obbligo d'insegnamento e di servizio prevede 340 ore per anno accademico, mentre le 240 ore d'insegnamento possono essere concentrate in un semestre. Ciò consente ai professori di svolgere inoltre anche altri compiti, non di rado dietro retribuzione, cosa prevista dalla legge e che spesso avviene, nonostante lo stipendio degli ordinari sia più alto di quello previsto in quasi tutti i paesi europei ${ }^{23}$. Un numero considerevole di parlamentari ancora oggi è contemporaneamente anche professore universitario (anche se quasi sempre in distacco) ${ }^{24}$.

Quinto: gli studenti. Questi pagano tasse universitarie e ricevono in cambio un insegnamento accademico di norma pessimo, che comprende ben poco oltre i corsi ordinari, in cui professori o ricercatori recitano dai loro libri di testo. L'insegnamento basato sulla ricerca è la grande eccezione. Del resto come potrebbe funzionare il sistema con università strapiene - l'università di Roma La Sapienza ha avuto per un certo periodo 120.000 studenti — , infrastrutture carenti e con il personale impegnato in molte altre faccende? La procedura tollera perciò una sorta di studio "esterno", in cui ci si incontra spesso per la prima volta in occasione dell'esame - quasi sempre orale. L'Italia da tempo forma, come molte altre nazioni dell'Europa meridionale, molte più persone di quante ne siano necessarie e la disoccupazione accademica è tradizionalmente alta, ma non aveva mai raggiunto, come oggi, la quota di oltre il 40 per cento dei laureati il che rappresenta anche un effetto della difesa a oltranza del diritto al lavoro, che conosce solo gli interessi di chi un impiego lo possiede già.

\section{L'Abilitazione scientifica nazionale}

Solo chi la supera può prendere parte in seguito a concorsi. L'Abilitazione scientifica nazionale è a sua volta una valutazione delle pubblicazioni e del loro rapporto con l'anzianità di servizio, che si tiene mediante bando pubblico teoricamente una volta l'anno ed è eseguita da commissioni specifiche organizzate per materia, composte da cinque membri, di cui uno obbligatoriamente straniero, e nominate ogni due anni. L'abilitazione non assegna, è bene sottolinearlo ancora una volta, né un impiego né fondi, bensì un attestato valido solo sei anni ${ }^{25}$. Le commissioni si riuniscono occasionalmente nella sede dell'u-

${ }^{23}$ Nel 1986 la retribuzione annua oscillava tra i 90.000 e i 170.000 marchi. Si veda Thomas Finkenstaedt, Teachers, in Rüegg (ed.), History of the University, cit., pp. 162-203, qui p. 184 sg.

${ }^{24}$ Dal 1948 raramente si è scesi sotto la soglia del 10 per cento dei deputati. Si veda Palermo, L'università dei baroni, cit., tab. a p. 42

${ }^{25}$ L'abilitazione scientifica nazionale aveva una validità di soli quattro anni. Questo dettaglio rinvia, insieme ad altri, al modello della Francia, dove un attestato simile dura quattro an- 
niversità che le coadiuva, in quell'occasione confrontano le loro valutazioni - ogni esaminatore deve giudicare per iscritto ogni candidato - , discutere controversie e casi limite e infine dare un voto su ogni singolo candidato. La procedura termina nel momento in cui i voti individuali e la deliberazione della commissione vengono pubblicati in rete in data prestabilita. Contro le decisioni negative i candidati possono fare ricorso al tribunale amministrativo, di cui molti si servono; se con successo, finora non è dato sapere, vista la nota lentezza della giustizia italiana ${ }^{26}$.

A questa nuova procedura si devono alcuni giudizi che hanno reso possibile questo contributo e che valgono sicuramente solo come istantanee. Chi si candida all'abilitazione deve fornire, accanto a un curriculum vitae completo, una lista delle pubblicazioni e, a seconda del posto di docenza ambito (associato o ordinario), dodici o diciotto file in formato pdf riguardanti le più recenti ricerche effettuate, che vengono messe a disposizione dei membri della commissione in un database protetto da password. Ciò rappresenta la base materiale per le seguenti considerazioni.

Nella disciplina della Storia contemporanea, oggetto in passato in Italia di accese controversie e sottoposta ai tentativi d'influenza da parte ministeriale ${ }^{27}$ nello specifico essa coincide con quella che in tedesco è definita "Neueste Zeit" 28 - non meno di 541 persone hanno consegnato la propria documentazione in occasione del primo passaggio nel 2012-2013, 425 per un posto di associato e 116

ni, a ogni modo con la possibilità di richiedere un prolungamento. Il limite rigoroso è avulso dal contesto e provoca soltanto un ulteriore dispendio di energie sia per i candidati sia per gli esaminatori, dal momento che si può presentare domanda addirittura per anni ancora oltre il limite dell'età pensionabile.

${ }^{26}$ Le cifre sui procedimenti civili si trovano in Bianco, Napolitano, Why the Italian Administrative System, cit., p. $550 \mathrm{sg}$.

${ }^{27}$ Dettagli si trovano in Lutz Klinkhammer, Novecento statt Storia contemporanea? Überlegungen zur italienischen Zeitgeschichte, in Alexander Nützenadel, Wolfgang Schieder (hrsg.), Zeitgeschichte als Problem. Nationale Traditionen und Perspektiven der Forschung in Europa, Göttingen, Vandenhoeck \& Ruprecht, 2004, pp. 107-127, come in Cavazza, Die Neueste Geschichte, cit., in part. p. $92 \mathrm{sg}$.

${ }^{28}$ Ciò che rientra nella Storia contemporanea per il processo di abilitazione è stato definito dal decreto ministeriale n. 336 del 29 luglio 2011 in questi termini: "Il settore si interessa all'attività scientifica e didattico-formativa nei campi che riguardano le competenze relative agli ultimi due secoli a partire dagli eventi politici tardo settecenteschi che propongono i temi universali dell'autodeterminazione e della cittadinanza (rivoluzione americana e rivoluzione francese). Il settore ricomprende anche la Storia dell'Europa orientale nell'età contemporanea, che si caratterizza per la specificità geografica e storico-culturale soprattutto a partire dall'età delle rivoluzioni. Si caratterizza per l'attenzione ai fenomeni di interdipendenza mondiale, massificazione e accelerazione dei processi socio-economici. In quanto indagine volta alla chiarificazione del nostro tempo, sviluppa l'intreccio tra storia e memoria, storia di genere, fenomeni politico-istituzionali, religiosi, socio-culturali, militari. Comprende inoltre studi relativi alla metodologia, alla storiografia e alla didattica del periodo considerato", si veda http://attiministeriali.miur.it/ anno-2011/luglio/dm-29072011 (ultimo accesso 7 agosto 2016), Allegato B, p. 48. Ai fini pratici basta anche questa stentata definizione. 
per quello di ordinario, a evidente testimonianza dell'enorme ingorgo di personale ${ }^{29}$. Nella seconda tornata del 2013-2014 in entrambe le categorie vi erano appena 98 candidati, 77 per il posto di associato e 21 per quello di ordinario ${ }^{30}$. Si può affermare a buon diritto che in questi numeri sia rappresentato l'insieme delle nuove leve accademiche e anche di più, come si mostrerà a breve. Ciò rende una comparazione con la Germania in senso lato impossibile, dal momento che nella Repubblica federale non esiste alcuna forma di rilevamento comparabile; nessuno, neppure i capi gruppo di selezione e d'assegnazione (Fachkollegium) della Dfg (Deutsche Forschungsgemeinschaft) per le scienze storiche, ha una visione d'insieme anche lontanamente simile del campo professionale di quegli storici che si trovano al di sotto della categoria dei professori ordinari e che attualmente cercano di migliorare la propria posizione professionale. Così forse si potrebbe definire il gruppo qui preso in esame.

Qui di seguito si elencheranno concretamente le tipologie di categorie professionali.

A proposito della tabella 1 ci sono alcuni punti da chiarire. I classici aspiranti ai posti di professore universitario sono i ricercatori, assunti fino al 2010 prevalentemente a tempo indeterminato e più recentemente a tempo determinato, i quali costituiscono l'ossatura del corpo scientifico delle università italiane; i più anziani tra questi naturalmente non possiedono il titolo di dottore di ricerca (corrispondente all'inglese $\mathrm{PhD}$ o al tedesco Dr. phil.), introdotto con una legge solo nel $1980^{31}$. Assegnisti e borsisti, sostanzialmente dei post-dottorandi, beneficiano di un sostegno finanziario temporaneo alla ricerca, mentre i cultori della materia equivalgono alla lontana a dei collaboratori scientifici laureati, a ogni modo impiegabili, dal momento che il loro utilizzo è limitato all'organizzazione di lezioni, esami e simili; non sono pagati, ma si trovano a stretto contatto con le figure influenti. Del tutto insolita è stata la forte partecipazione di bibliotecari e insegnanti di scuola di tutti i livelli ${ }^{32}$, mentre gli archivisti, così come anche in Germania, tendono a considerarsi storici ricercatori. Che appartenenti ad altre categorie professionali lontane dalla ricerca scientifica presentino domanda per ottenere un posto di lavoro a tempo indeterminato come professore e quindi puntino all'abilitazione sarebbe in Germania del tutto incomprensibile, ma si ricollega al basso grado di professionalizzazione, alme-

${ }^{29} \mathrm{~A}$ sua volta reso ancor più chiaro se si prende in considerazione la somma complessiva di 59.193 candidati ammessi.

${ }^{30}$ Non c'è bisogno di approfondire le condizioni limite di una tale maratona valutativa anche per esaminatori esperti.

${ }^{31}$ Diversamente dalla Germania, ma in modo simile alla Francia, questo titolo viene conseguito o consegnato solo nella prospettiva di una ricerca.

${ }^{32}$ Esiste ancora in Italia (e in molti altri paesi) una permeabilità fra scuola e università, mentre in Germania carriere come quelle di Ernst Nolte, Andreas Hillgruber o Volker Reinhard, che hanno iniziato come insegnanti di scuola secondaria, sarebbe oggigiorno impensabile — anche perché nel frattempo gli insegnanti sono meglio pagati dei ricercatori scientifici, per non parlare delle più sicure prospettive lavorative. 
no dalla prospettiva tedesca, dei docenti universitari italiani ${ }^{33}$ : come già brevemente accennato, i professori italiani congiungono spesso il loro impiego accademico con molte altre attività, in particolare nel ramo del giornalismo, della politica e in tutte le funzioni del settore della cultura e di conseguenza anche all'inverso l'afflusso da altre sfere lavorative all'impiego di professore universitario ha una lunga tradizione. Inoltre fino a poco tempo fa non vi era alcun requisito vincolante, come l'abilitazione o altre forme di qualificazione ${ }^{34}$.

Tab. 1 - Candidati per posti da associato nel 2012 e 2013: rispettivamente 425 e 77 candidati

a) Ordinati per impieghi e attività

\begin{tabular}{l|r|r|r|r}
\hline Categorie & \multicolumn{2}{|c|}{2012} & \multicolumn{2}{|c}{2013} \\
\hline Ricercatori universitari & 169 & $39,1 \%$ & 20 & $25,9 \%$ \\
\hline Ricercatori presso istituti non universitari & - & - & 6 & $7,8 \%$ \\
\hline Assegnisti di ricerca & 32 & $7,5 \%$ & 13 & $16,8 \%$ \\
\hline Borsisti post-doc & 8 & $1,8 \%$ & - & - \\
\hline Cultori della materia & 16 & $3,6 \%$ & 4 & $5,2 \%$ \\
\hline Impiegati in istituti di ricerca stranieri & 30 & $7,0 \%$ & 6 & $7,8 \%$ \\
\hline (di cui grazie al Marie Curie Fellowship) & 5 & $1,2 \%$ & 1 & $1,2 \%$ \\
\hline Professori a contratto & 37 & $8,7 \%$ & 10 & $12,9 \%$ \\
\hline Bibliotecari, archivisti & 8 & $1,8 \%$ & 1 & $1,2 \%$ \\
\hline Insegnanti di ogni livello scolastico & 52 & $12,2 \%$ & 7 & $9,1 \%$ \\
\hline Giornalisti, addetti nell'editoria & 12 & $2,8 \%$ & 2 & $2,6 \%$ \\
\hline Impiegati e funzionari dell'amministrazione pubblica, altro & 27 & $6,1 \%$ & 4 & $5,2 \%$ \\
\hline Liberi professionisti e lavoratori autonomi & 9 & $2,1 \%$ & - & - \\
\hline Militari & 3 & $0,7 \%$ & - & - \\
\hline Pensionati & 1 & $0,2 \%$ & 2 & $2,6 \%$ \\
\hline $\begin{array}{l}\text { Disoccupati } \\
\text { (in parte con ridotti incarichi di ricerca) }\end{array}$ & 19 & $4,4 \%$ & 2 & $2,6 \%$ \\
\hline Jean Monnet Professors e professori associati di altro tipo & 2 & $0,4 \%$ & - & - \\
\hline Tot. & 425 & $100 \%$ & 77 & $100 \%$ \\
\hline
\end{tabular}

${ }^{33}$ Un approfondimento a tal proposito in Marcel vom Lehn, Westdeutsche und italienische Historiker als Intellektuelle? Ihr Umgang mit Nationalsozialismus und Faschismus in den Massenmedien (1943/45-1960), Göttingen, Vandenhoeck \& Ruprecht, 2012, cap. I.

${ }^{34}$ In questo modo l'appena venticinquenne giornalista e (in seguito) politico Giovanni Spadolini fu chiamato ad assumere nel 1960 la prima cattedra italiana di Storia contemporanea all'Università di Firenze. 
b) Ordinati per gruppi

\begin{tabular}{l|r|r|r|r}
\hline Categorie & \multicolumn{2}{|c|}{2012} & \multicolumn{2}{|c}{2013} \\
\hline 1) Direttamente occupati nelle università & & & & \\
\hline Collocati in università italiane e straniere & 199 & $46,5 \%$ & 32 & $29,8 \%$ \\
\hline In sottostrutture universitarie & 56 & $13,1 \%$ & 17 & $22,0 \%$ \\
\hline Con incarichi di insegnamento & 37 & $8,7 \%$ & 10 & $12,9 \%$ \\
\hline Jean Monnet Professors, professori associati & 2 & $0,4 \%$ & - & - \\
\hline Tot. parziale & 294 & $69 \%$ & 59 & $77 \%$ \\
\hline 2) Vicini al settore della ricerca & & & & \\
\hline Bibliotecari, archivisti & & & & \\
\hline Insegnanti di ogni livello scolastico & 8 & $1,8 \%$ & 1 & $1,2 \%$ \\
\hline Tot. parziale & 52 & $12,2 \%$ & 7 & $9,1 \%$ \\
\hline & 60 & $14 \%$ & 8 & $10 \%$ \\
\hline 3) Altro & & & & \\
\hline
\end{tabular}

Non tutti ma comunque la maggior parte di questi candidati riusciva a soddisfare quantomeno la gran quantità di requisiti richiesti, e ciò è da porre in stretta relazione con la tradizione della cultura umanistica in Italia, di cui molto più che altrove sono propri lo scrivere e il pubblicare, entrambi incentivati con decisione. Gli storici disoccupati sono con il 4 e il 3 per cento nettamente sottorappresentati; altri disoccupati si celano sicuramente tra i cultori, nel personale con incarichi di insegnamento, tra i giornalisti e probabilmente anche tra i lavoratori autonomi. Riunendo i gruppi professionali emerge un'immagine chiara ma sempre molto diversa da quella della Germania e d'altronde nel 2013 dissimile da quella dell'anno precedente: la componente degli occupati direttamente nelle università o in professioni vicine alla ricerca cresce e ciò vuol dire soltanto che la rosa dei canditati è stata in parte sfoltita, esaurendosi la riserva dei candidati estranei alla ricerca ${ }^{35}$. La riduzione del numero dei ricercatori e il proporzionale raddoppiamento dei borsisti è ad ogni modo un segnale d'allarme.

Come prevedibile il campo di coloro che entrano in ballo per un posto da ordinario (tab. 2) è più nettamente professionalizzato. Liberi professionisti, indipendenti e storici in uniforme sono del tutto assenti e anche la quota delle professioni qui raggruppate come attinenti alla ricerca, confrontata con la categoria degli associati, decresce leggermente nel 2012 e arriva a zero nel 2013. Il

\footnotetext{
${ }^{35}$ Sempre che i respinti non tentino di candidarsi nuovamente nel 2014 o nel 2015, dal mo-
} mento che la ricandidatura è preclusa loro solo per un anno. 
Tab. 2 - Candidati per posti da ordinario nel 2012 e 2013: rispettivamente 116 e 21 candidati

a) Ordinati per impieghi e attività

\begin{tabular}{l|r|r|r|r}
\hline Categorie & \multicolumn{2}{|c|}{2012} & \multicolumn{2}{c}{2013} \\
\hline Professori associati & 51 & $44,0 \%$ & 9 & $42,8 \%$ \\
\hline Professori ordinari in università non statali & 1 & $0,9 \%$ & - & - \\
\hline Ricercatori & 31 & $26,7 \%$ & 7 & $33,3 \%$ \\
\hline Ricercatori presso istituti non universitari & 3 & $2,6 \%$ & 2 & $9,5 \%$ \\
\hline Assegnisti & 2 & $1,7 \%$ & 1 & $4,7 \%$ \\
\hline Professori a contratto & 5 & $4,3 \%$ & - & - \\
\hline Impiegati in università o istituti di ricerca stranieri & 4 & $3,4 \%$ & 1 & $4,7 \%$ \\
\hline Marie Curie Fellowship & 1 & $0,9 \%$ & - & - \\
\hline Insegnanti di ogni livello scolastico & 6 & $5,2 \%$ & - & - \\
\hline Archivisti & 1 & $0,9 \%$ & - & - \\
\hline Giornalisti & 4 & $3,4 \%$ & - & - \\
\hline Militari & 2 & $1,7 \%$ & - & - \\
\hline Indipendenti & 2 & $1,7 \%$ & - & - \\
\hline Altro & 3 & $2,6 \%$ & 1 & $4,7 \%$ \\
\hline Tot. & 116 & $100 \%$ & 21 & $100 \%$ \\
\hline
\end{tabular}

b) Ordinati per gruppi

\begin{tabular}{l|r|r|r|r}
\hline Categorie & \multicolumn{2}{|c|}{2012} & \multicolumn{2}{c}{2013} \\
\hline 1) Direttamente occupati nelle università & & & & \\
\hline $\begin{array}{l}\text { Collocati in università italiane e straniere e in istituti di ricerca } \\
\text { extrauniversitari }\end{array}$ & 90 & $77,6 \%$ & 20 & $95,2 \%$ \\
\hline In sottostrutture universitarie & 2 & $1,7 \%$ & - & - \\
\hline Con incarichi di insegnamento & 5 & $4,3 \%$ & - & - \\
\hline Tot. parziale & 97 & $84 \%$ & 20 & $95 \%$ \\
\hline & & & & \\
\hline 2) Vicini al settore della ricerca & & & & \\
\hline Bibliotecari, archivisti & 1 & $0,9 \%$ & - & - \\
\hline Insegnanti di ogni livello scolastico & 6 & $5,2 \%$ & - & - \\
\hline Tot. parziale & 7 & $6 \%$ & - & - \\
\hline & & & & \\
\hline 3) Altro & 12 & $10 \%$ & 1 & $5 \%$ \\
\hline
\end{tabular}


classico aspirante al posto di lavoro di professore ordinario è il professore associato, immediatamente seguito comunque dalla categoria dei ricercatori. Insieme questi due gruppi hanno quasi il monopolio delle candidature nel 2013. Il gruppo dei ricercatori desiderosi di tornare dall'estero rimane trascurabile; le basse cifre confermano quanto già detto sullo scarso richiamo del sistema scientifico italiano.

\section{Specificità tedesche e italiane}

Prima di entrare nei dettagli, è consigliabile un breve sguardo a quello che, della disciplina storica tedesca, susciterebbe la curiosità di un italiano, a partire dai diversi modi di affrontare l'ampio campo disciplinare. Come si può ben vedere a ogni edizione dell'Historikertag ${ }^{36}$, nelle scienze storiche rientra decisamente molto di più di quanto emerga dai Dipartimento di storia, con le loro cattedre definite principalmente in senso cronologico. A loro volta questi Dipartimenti fanno parte prevalentemente di facoltà umanisticheo di quelle che le hanno sostituite. In Italia invece vi sono Dipartimenti di storia sia nelle Facoltà di Lettere sia in quelle di Scienze politiche, quest'ultime introdotte all'epoca del fascismo (cosa che spiega la posizione di forza della storia politica), occasionalmente anche in quelle orientate alla formazione degli insegnanti (Magistero, poi Scienze della formazione). Raramente però al di fuori di queste esistono sottodiscipline consolidate. Agli italiani non sfuggirebbe inoltre neppure il fatto che la storia agraria, così importante in Italia, a nord delle Alpi si trova istituzionalmente relegata in un angolo (e perciò prevalentemente gestita come attività secondaria da storici generalisti), che la storia della tecnica e della scienza dopo un lungo periodo in ombra ascende nel segno del cultural turn alla storia generale, ma che di norma, al pari della storia economica, non rientrano nei Dipartimenti di storia; soprattutto però noterebbero che la storia regionale del XIX e XX secolo, eccettuato il caso della Baviera, rappresenta un settore marginale poco attrezzato, di cui si occupano soprattutto archivisti e commissioni storiche locali ampiamente rappresentate da storici non professionali, mentre vi sono diverse cattedre di storia della chiesa nelle facoltà di teologia, da tempo invece non presenti nell'insegnamento universitario pubblico in Italia.

D'altra parte in Germania, almeno fino alla riforma che ha introdotto il cosiddetto "processo di Bologna", i percorsi professionali fissati in maniera molto

${ }^{36} \mathrm{Si}$ tratta di una riunione biennale degli storici tedeschi di tutte le discipline (NdT). Questo genere di manifestazione non ha tra l'altro un equivalente in Italia, né vi è lì un'associazione generale di tutti gli storici. A risultare organizzati sono sempre gli interessi particolari. Così fu fondata nel 1989 la Società italiana delle storiche (Sis), a cui seguì nel 1990 la Società italiana per lo studio della storia contemporanea (Sissco). Dal 2011 esiste un'organizzazione di storici contemporanei per l'ambito culturale tedesco, la Società italiana per la storia contemporanea dell'area di lingua tedesca (Siscalt) e via dicendo. 
meno rigida - fino a pochi decenni fa ciò rappresentava la quint'essenza della "libertà accademica" difesa anche dagli studenti - e questo, grazie soprattutto alla configurazione delle cattedre di storia su base prettamente cronologica, permettevano ai possessori delle cattedre di operare in ogni ambito e con chiunque volessero, tanto nella ricerca quanto nell'insegnamento, purché si avesse un'attinenza con il passato. Questo è all'origine della grande estensione di contenuti, recentemente messa a rischio, in cui può spaziare un professore di storia tedesco a seconda della sua volontà e che non trova corrispettivi al di fuori dell'area di lingua tedesca. In Italia l'impegno nell'insegnamento è molto minore, sia quantitativamente che qualitativamente, dal momento che i curricula permettono la riproposizione, ogni anno, del programma di studi approvato in origine. Ciò va a vantaggio della produttività pubblicistica, di cui si parlerà in seguito.

Anche altri aspetti sono stati regolati di recente in Italia in modo del tutto diverso dalla Germania. Il già citato ministro della Pubblica istruzione Luigi Berlinguer, rimasto in carica tra il 1996 e il 2000, quindi sia sotto il governo Prodi che quello del suo successore Giuliano Amato, si prefisse una serie di riforme nel settore universitario. Tra queste si annovera la fallimentare riforma delle procedure per i bandi e le assunzioni e quella del 1998 per il miglioramento delle scuole di dottorato, che cercava di seguire il modello americano. Da allora la legge non permette alcun dottorato al di fuori di tali scuole di dottorato, di cui perciò è munito quasi ogni dipartimento. Si è ammessi attraverso bando e una parte degli ammessi ottiene una borsa, mentre i restanti devono pagare una tassa. Ogni scuola ha ovviamente un tema, che può essere, a seconda delle università, molto generico o molto specifico, ma può anche presentare un'impostazione particolarmente esigente sul piano delle premesse teoriche ${ }^{37}$. Nel primo anno si insegna e si svolgono corsi, mentre nei successivi due anni la ricerca autonoma ha la priorità, dal momento che nessun dottorato può durare oltre i tre anni. Le conseguenze per la scienza sono rilevanti, dal momento che con questa gestione del personale di ricerca diretta e orientata alla visibilità, all'efficienza e di conseguenza al controllo diminuisce la possibilità di approcci individuali e originali alla ricerca, oltre alla formulazione di quesiti in chiavi non ortodosse. Anche in Germania, grazie all'abbondante flusso di fondi dall'esterno, la tendenza va nella stessa direzione; tuttavia per fortuna della ricerca sono (ancora per molto?) possibili dottorati indipendenti in Lettere e nelle Scienze sociali; in tal modo si agevola il coesistere dei due sistemi anche nella stessa università e, più in generale, il permanere di una sana competizione.

\footnotetext{
${ }^{37}$ Esempi della prima variante sono Scienze storiche ad Alessandria o Storia contemporanea a Roma Tre, oppure Scienze giuridiche, storiche e sociali a Siena. Nel secondo caso vi sono titoli come Ceti dirigenti e potere pubblico nella storia d'Italia contemporanea alla Sapienza di Roma o Storia dei partiti e dei movimenti politici nell'Europa del XIX e XX secolo a Bologna. Infine la terza variante presenta temi come Teoria e storia della modernizzazione e del cambiamento sociale in età contemporanea a Siena, Crisi e trasformazione della società contemporanea a Torino o Pensiero politico e istituzioni nelle società mediterranee a Catania.
} 
Al di là delle istituzioni appartengono alla diversa cultura accademica della storia naturalmente anche una certa visione dei ruoli, specifici sistemi di valori e peculiarità epistemiche. Queste ultime, con uno sguardo comparato alla produzione scientifica nei paesi confinanti, presentano evidenti differenze sotto molti punti di vista. Qui si farà brevemente riferimento a tre di questi elementi distintivi.

Innanzitutto l'importanza accademica della teoria in Germania. Il celebre saggio di Koselleck sul bisogno di teoria della scienza storica ${ }^{38}$ non trova in Italia alcun pendant. Anche se questa esigenza spesso non viene sufficientemente onorata, da almeno tre o quattro decenni nessuno storico tedesco può espressamente pensare che basti scrivere pragmaticamente a braccio. Oggigiorno i ricercatori si sentono più che in passato nella necessità di fornire motivazioni teoriche per la scelta dei temi e per l'impalcatura della ricerca. In Italia ciò avviene invece più raramente.

Ciò si ricollega in secondo luogo alla funzione esemplare della scienza storica anglosassone; da tempo questa è garantita in Germania anche sul piano istituzionale. Nuovi filoni della ricerca che emergono negli Usa in Germania trovano immancabilmente attenzione e riproposizioni (se ciò sia sempre legittimo è un altro discorso), mentre la Francia, cui tradizionalmente la storiografia italiana è legata, spesso accoglie i ricercatori italiani (molti candidati detengono titoli francesi fino a quello di Maître de conférences), ma nella storia contemporanea offre loro sul piano metodologico e tematico ben pochi spunti ${ }^{39}$. La conseguenza è che in Germania ha avuto luogo una "dinamica delle svolte" che talvolta ha assunto addirittura un carattere costrittivo, a cui in Italia si contrappone una palese staticità, che trova la sua espressione nel dominio di una forma di racconto oggettivo della storia politica.

Un terzo elemento infine è la discussione, partita dopo la riunificazione tedesca, se non prodotta direttamente da questa, sulle fondamenta e sull'orientamento di fondo della storia contemporanea come disciplina. In proposito il suggerimento di Hockerts, di servirsi della storia contemporanea come di un'introduzione alle problematiche del presente ${ }^{41}$, si è rivelato particolarmente stimolante. Con ciò temi come la trasformazione dei valori, gli schemi di per-

${ }^{38} \mathrm{Si}$ veda Reinhart Koselleck, Über die Theoriebedürftigkeit der Geschichtswissenschaft, in Id., Zeitschichten. Studien zur Historik, Frankfurt am Main, Suhrkamp, 2000, pp. 298-316.

${ }^{39}$ Rainer Hudemann riconduce parte del problema allo strapotere della scuola degli Annales. Si veda Rainer Hudemann, Histoire du Temps présent in Frankreich. Zwischen nationalen Problemstellungen und internationaler Öffnung, in Nützenadel, Schieder (a cura di), Zeitgeschichte als Problem, cit., pp. 175-200, in part. p. 187.

${ }^{40}$ Ironico quanto ricco di spunti è in proposito Christoph Conrad, Die Dynamik der Wenden. Von der neuen Sozialgeschichte zum cultural turn, in Jürgen Osterhammel, Dieter Langewiesche, Paul Nolte (hrsg.), Wege der Gesellschaftsgeschichte, Göttingen, Vandenhoeck \& Ruprecht, 2006, pp. 133-160.

${ }^{41}$ Cfr. Hans Günter Hockerts, Zeitgeschichte in Deutschland. Begriff, Methoden, Themenfelder, "Historisches Jahrbuch", 1993, n. 111, pp. 98-127. 
cezione e comportamento o la globalizzazione sono entrati nel campo visivo degli storici, costringendoli a una storia contemporanea vicina all'attuale e in grado di confrontarsi criticamente con altre discipline orientate al presente. $\mathrm{Si}$ tratta dell'esatto opposto del modello di storia contemporanea condotto in maniera preponderante in Italia, il quale resta concentrato sul biennio 1943-1945 e sugli anni Settanta, pone al centro partiti, correnti politiche e uomini politi$\mathrm{ci}^{42}$ e rarissimamente analizza le crisi che da tre decenni attanagliano il Paese. Probabilmente ciò appare troppo pericoloso soprattutto ai ricercatori, nonostante le fratture politico-culturali fra destra e sinistra si siano ormai nettamente attenuate. Per lo stesso motivo emergono delle mancanze anche nella storia della storiografia, dove si hanno biografie di storici abbozzate in maniera agiografica. La curiosità intellettuale e metodologica, legata a una dose di coraggio e forse addirittura al piacere per la provocazione non è certamente la regola della storiografia tedesca e forse proprio per questo la Società degli storici ha premiato nel 2008 una tesi di dottorato sulla prostituzione maschile ${ }^{43}$. Di libri del genere, anche solo alla lontana, non c'è traccia fra quelli sottoposti alla commissione per l'abilitazione scientifica nazionale.

\section{Contenuti principali}

Quali sono allora gli ambiti tematici principali ${ }^{44}$ ? Seguendo l'ordine cronologico si parte dall'epoca della rivoluzione francese. Non è un caso, dal momento che, a seguito della liberazione della disciplina storica dagli stereotipi nazionali, si è visto in essa il passo decisivo per coniare una coscienza nazionale. Ancor di più stupisce dunque che di quest'epoca non si occupi nessuna delle ricerche. Lo stesso vale per Napoleone e anche il Risorgimento, con poche eccezioni $^{45}$, scompare dal campo visivo degli studiosi, nonostante questo, grazie

${ }^{42}$ Il fatto che il caso Moro distacchi di molto ogni altro argomento mette in luce da un lato il carattere enigmatico e traumatico di tale vicenda, dall'altro i limiti autoimposti della riflessione storico-politica, perché la caduta della cosiddetta prima repubblica a seguito di un'ondata di affari di corruzione e finanziamenti illeciti ai partiti — davvero un problema di primo piano della storia contemporanea - non ha niente a che fare con l'omicidio Moro.

${ }^{43} \mathrm{Si}$ veda Martin Lücke, Männlichkeit in Unordnung. Homosexualität und männliche Prostitution im Kaiserreich und Weimarer Republik, Frankfurt am Main-New York, Campus Verlag, 2008.

${ }^{44}$ È fuori discussione cercare di fornire un'immagine in scala della produzione dei 631 candidati. Perciò ho messo insieme per entrambi i cicli e per entrambi i gruppi di candidati una selezione (il 10 per cento dell'intera schiera distribuito ugualmente fra ammessi e bocciati). A volte faccio riferimento anche ad altre persone.

${ }^{45}$ Qui vale soprattutto il contributo di Arianna Arisi Rota; si veda Arianna Arisi Rota, Monica Ferrari, Matteo Morandi, (a cura di), Patrioti si diventa. Luoghi e linguaggi di pedagogia patriottica nell'Italia unita, Milano, FrancoAngeli, 2009, e anche Arianna Arisi Rota, Discovering Politics: Action and Recollection in the First Mazzinian Generation, in Silvana Patriarca, Lucy Riall (eds.), The Risorgimento Revisited. Nationalism and Culture in Nineteenth-Century Italy, Basingstoke, Palgrave Macmillan, 2012, pp. 77-96. 
alla notevole reinterpretazione operata da Banti nel segno del cultural turn, sia stato liberato da un ruolo specifico finora difeso a spada tratta e si sia aperto alla comparazione internazionale ${ }^{46}$. Salta all'occhio la preferenza per la storia della memoria, divenuta ormai di moda, ma qui le cose non sono tanto diverse dalla Germania, in quanto anche lì la fondazione dello stato nazionale trova ben poco interesse presso i ricercatori più giovani. Fra i ricercatori italiani, al di là di alcune sintesi generali, il XIX secolo sul piano della storia dello Stato non trova più alcuno spazio. È solo con la crisi a cavallo fra i due secoli e con $\mathrm{i}$ mutamenti dell'epoca delle riforme di Giolitti che si riaccende un certo interesse. Lo stesso vale per la Prima guerra mondiale, i cui studiosi si contano sulle dita di una mano e hanno spesso come oggetto di studio la storia delle esperienze (lettere dal fronte).

Tra gli argomenti principali della ricerca sulla storia contemporanea vi è, come da previsione, il fascismo. Il 12 per cento degli autori inserisce la parola nel titolo del proprio libro; una percentuale di gran lunga maggiore si occupa principalmente di altri temi, che si estendono tuttavia anche al fascismo. I temi più frequenti e rappresentati in ricerche dettagliate sono quelli sulla presa del potere, sul controllo dell'opinione pubblica, soprattutto però sulla repressione, e qui a margine anche sulla collaborazione italo-tedesca, e ovviamente sulla Resistenza, mito di fondazione della Repubblica a lungo sovraccaricato e affrontato in Italia nel segno delle due parole chiave Resistenza e Antifascismo. Il carattere criminale del regime mussoliniano ha inoltre sostituito la leggenda degli "italiani brava gente", diversamente da quanto è accaduto nell'opinione della maggior parte degli italiani. In proposito la ricerca si è liberata anche delle minimizzazioni di Renzo De Felice, ma è ancor di più sottoposta all'influenza dell'interpretazione storico-spiritualistica del suo allievo Gentile ${ }^{47}$. La Repubblica sociale italiana e il fascismo dopo il 1945 rappresentano oggi come in passato un'eccezione. Complementari all'opera pionieristica di Thomas Schlemmer sono presenti anche due lavori sui prigionieri di guerra italiani in Unione Sovietica ${ }^{48}$.

Spiccano invece, perché di tutt'altra natura, alcune opere incentrate sul razzismo. Da un lato queste, con lo sguardo rivolto a Telesio Interlandi e a Julius Evola e al loro obiettivo di valorizzare in maniera ultimativa l'Italia sul piano razziale, accantonano apertamente e definitivamente l'impostazione discolpante di De Felice e Denis Mack Smith; dall'altro la storia della ricerca di un "uomo nuovo", da Lombroso fino al 1943, colma le lacune rispetto alla situazione in

\footnotetext{
${ }^{46} \mathrm{Si}$ veda Alberto M. Banti, La nazione del Risorgimento. Parentela, santità e onore dell'Italia unita, Torino, Einaudi, 2000.

${ }^{47}$ Qui basta citare Emilio Gentile, Il culto del littorio. La sacralizzazione della politica nell'Italia fascista, Roma-Bari, Laterza, 1993.

${ }^{48} \mathrm{Si}$ veda Thomas Schlemmer, Die Italiener an der Ostfront 1942/43. Dokumente zu Mussolinis Krieg gegen die Sowjetunion, München, Oldenbourg, 2005.
} 
altri Paesi e documenta di una discussione eugenetica, la quale rappresentava sì un progetto di modernità, ma che in Italia, grazie al grande influsso della chiesa, ebbe tuttavia risvolti molto meno radicali rispetto anche ad altri paesi cattolici ${ }^{49}$. A ogni modo la sempre presente ritrosia italiana nel porre il fascismo in relazione con la modernità ${ }^{50}$, viene qui finalmente trasferita sul piano essenziale della riflessione teorica; ciò vale anche per un altro libro sulle innovazioni nel campo delle politiche sociali - e sui loro limiti — nel caso dell'organizzazione assistenziale a Torino ${ }^{51}$.

L'altro dei due punti centrali è la storia dell'Italia postbellica. Nel campione preso in esame si tratta di una quota dal trenta al quaranta per cento totale dei titoli. Predominano, come già detto, opere sui partiti politici e su figure di comando rispetto alle istituzioni e ai processi storici, estendendosi a temi come la protesta giovanile e ovviamente il terrorismo, che funestò l'Italia molto più di qualsiasi altro paese occidentale. Vi sono certamente anche titoli sull'identità nazionale, sui simboli del miracolo economico, come la Vespa, sulla storia dei trasporti e delle città, ma con poche eccezioni le ricerche si fermano agli anni Settanta ${ }^{52}$. Per una disciplina legata a filo doppio alla storia politica ciò si spiega anche con il fatto che i documenti statali, anche per quello stesso decennio, semplicemente non sono ancora accessibili alla ricerca. Ma una storia contemporanea come premessa del presente necessita solo in seconda istanza degli archivi pubblici. In primo luogo fornisce strumenti per l'orientamento nel qui e nell'oggi, e anche in Italia essa dovrebbe essere altro dall'analisi di partiti decaduti o di uomini politici morti da tempo. Il tracollo in atto da tre decenni dell'Italia in così tanti ambiti, la fine della cosiddetta prima repubblica, il passaggio da paese di emigranti a paese d'immigrazione e soprattutto l'avvento dell'Italia nel mondo postcoloniale - di tutto ciò nella ricerca storiografica, almeno in quella delle due annate dei concorsi, non vi è alcuna traccia. Il compito di analizzare il fenomeno Berlusconi resta nelle mani di professori ordinari come Paul Ginsborg ${ }^{53}$ e del resto manca del tutto un qualsiasi sguardo sto-

${ }^{49}$ Si veda Francesco Cassata, "La Difesa della razza". Politica, ideologia e immagine del razzismo fascista, Torino, Einaudi, 2008; Id., Building the New Man. Eugenics, Racial Science and Genetics in Italy, 1912-1975, Budapest-New York, Central European University Press, 2011. Cassata ha scritto anche opere su Corrado Gini e Julius Evola e un libro ricco di spunti sul modo in cui i comunisti e altri in Italia hanno trattato il "caso Lysenko"; si veda Id., Il fascismo razionale. Corrado Gini tra scienza e politica, Roma, Carocci, 2006; Id., A destra del fascismo. Profilo politico di Julius Evola, Torino, Bollati Boringhieri, 2003, e Id., Le due scienze. Il "caso Lysenko" in Italia, Torino, Bollati Boringhieri, 2008.

${ }^{50} \mathrm{Si}$ rimanda all'ormai classico stupore suscitato dall'opera del marxista inglese Tim Mason, Italy and Modernization. A Montage, "History Workshop", 1988, n. 25/1, pp. 127-147.

${ }^{51} \mathrm{Si}$ veda Silvia Daniela Inaudi, A tutti indistintamente. L'Ente Opere Assistenziali nel periodo fascista, Bologna, Clueb, 2008.

${ }_{52}^{5}$ Le opere generali ovviamente vanno oltre, ma non si fondano su una ricerca propria e non svolgono quindi alcun ruolo per la valutazione compiuta in questa sede.

${ }_{53}$ Come esempio si veda Paul Ginsborg, Ambizioni patrimoniali in una democrazia mediati$c a$, Torino, Einaudi, 2003. 
riografico a sostegno di un'analisi del tempo presente e, conseguentemente, dei tentativi di risanamento della sfera politica. Resta vana la ricerca di libri indirizzati a un'analisi di autocomprensione intellettuale successiva alla guerra e al tracollo militare, quindi molto prima dei confini imposti dai tempi d'interdizione degli archivi ${ }^{54}$.

Fra gli altri temi emergono quantitativamente, se si esclude la forte presenza della storia locale e regionale, sulla quale si tornerà in seguito, la storia delle donne e la storia di genere ${ }^{55}$, la storia della chiesa e delle religioni in senso lato e, come facilmente prevedibile, la storia dell'emigrazione. Questa rientra da tempo fra le tematiche notoriamente classiche. Fornisce un contributo all'autocomprensione nazionale e quindi è molto ben organizzata, naturalmente non soltanto entro i confini italiani, in alcuni casi può contare sul sostegno finanziario della politica, si serve inoltre di numerosi istituti di qualità molto diversa e di alcune riviste specializzate ${ }^{56}$. Un problema, comunque non limitato alla sola Italia sta nel fatto che queste tematiche spesso sono elaborate da persone "coinvolte". Ciò non va a beneficio di queste sottodiscipline, poiché motivi identitari nel lavoro di ricerca scientifica sono immancabilmente limitanti e solo in casi eccezionali ragionati.

\section{I criteri di ammissione e le consuetudini accademiche}

Le disposizioni per la domanda di ammissione a una cattedra in Germania prevedono la presentazione dei certificati (per il dottorato e l'abilitazione o per prestazioni scientifiche riconosciute di pari grado). Solo rigorosamente, in un secondo momento, entrano in gioco monografie e successivamente anche altre pubblicazioni e, in tempi più recenti, indicazioni sull'esperienza nell'insegnamento e nell'ottenimento di fondi per la ricerca. Nel caso dell'Abilitazione

${ }^{54}$ Il pensiero va a tesi di dottorato come quelle di Dirk van Laak, Gespräche in der Sicherheit des Schweigens. Carl Schmitt in der politischen Geistesgeschichte der frühen Bundesrepublik, Berlin, Akademie-Verlag, 1993, e quella di Jens Hacke, Philosophie der Bürgerlichkeit. Die liberalkonservative Begründung der Bundesrepublik, Göttingen, Vandenhoeck \& Ruprecht, 2006.

${ }^{55} \mathrm{Nel}$ caso della storia di genere si annovera accanto alla già citata Silvia Inaudi anche Silvia Salvatici con il suo notevole libro Contadine dell'Italia fascista. Presenze, ruoli, immagi$n i$, Torino, Rosenberg \& Sellier, 1999, e l'opera di Patrizia Guarnieri sulle violenze su donne e bambini tra XIX e XX secolo. Tra i più recenti articoli di quest'ultima si ricordano: L'incesto scandaloso: legge e mentalità nell'Italia unita, "Passato e presente", 2003, n. 58, pp. 45-68, Id, Un piccolo essere perverso. Il bambino nella cultura scientifica italiana fra Otto e Novecento, "Contemporanea", 2006, n. 9, pp. 253-284, e non da ultimo Id, Men Committing Female Crime: Infanticide, Family and Honor in Italy 1890-1981, "Crime, Histoire \& Sociétés", 2009, n. 13, pp. $41-54$.

${ }^{56} \mathrm{Cfr}$. la rivista "Altreitalie. Rivista internazionale di studi sulle migrazioni italiane nel mondo", fondata nel 1989 e operante con un sistema di peer review. 
scientifica nazionale in Italia ciò non è possibile, in quanto il dottorato di ricerca, come già ricordato, viene conferito solo dal 1980 e perciò escluderebbe candidati più anziani, mentre il certificato di abilitazione è in concreto lo scopo finale dell'intero procedimento. Per questo ci si aiuta in altro modo. I parametri stabiliti dal ministero sono di natura quantitativa. Fanno riferimento, come già accennato, a un numero minimo di libri e saggi di pubblicazione recente, distinti in base al tipo di cattedra a cui si aspira. Al fine di non porre in una posizione di svantaggio i più giovani rispetto a chi si trovi in servizio da più tempo, è stata introdotta un'età accademica, vale a dire un indicatore per calcolare la produttività in rapporto al tempo trascorso. Al più tardi qui entra in gioco la produttività scientifica ma significativamente in una forma conforme alla valutazione, quindi qualificante, con la conseguenza inevitabile che la logica della produzione scientifica viene ignorata e spesso addirittura contrastata ${ }^{57}$. "Normalizzazione" e standardizzazione sono all'ordine del giorno (non solo in Italia) e perciò la banca dati dei documenti allegati alle domande contiene per ogni persona tre valori computati sulla base delle pubblicazioni totali fornite, chiamati mediane, che dovrebbero aiutare nella valutazione. Queste mediane riportano per i candidati a professore associato i valori 1,71 per le monografie, 11,5 per altri scritti non autonomi e 0 per quanto riguarda opere all'interno di riviste con peer review; per l'ordinario invece i valori sono 2,16 e di nuovo 0 . La commissione da un lato era autorizzata a prevedere delle eccezioni a queste prescrizioni standardizzate, seguendo argomenti di carattere qualitativo, e in parte vi ha fatto anche ricorso; dall'altro lato però non ha potuto fare che inasprire i valori quantitativi ${ }^{58}$. Sono così emersi, inevitabilmente, atteggiamenti specifici profondamente radicati nel substrato culturale di questo paese sul tema della "produttività". Nella percezione italiana non basta evidentemente un secondo libro, come altrove richiesto, ma ci deve essere "qualcosa di più". Che ciò non abbia comportato difficoltà per i candidati è la conseguenza delle consuetudini accademiche. La produttività di storici e storiche italiani è in generale più alta dei colleghi tedeschi; quarantenni spesso esibiscono già quattro libri, nonostante il dottorato di ricerca venga perlopiù conseguito non prima dei trent'anni ${ }^{59}$. Pre-

${ }^{57}$ I blog sono di conseguenza pieni di rimostranze e di assurdi modelli di conteggio. Invece di fare diversi esempi si rimanda a http://www.roars.it/online/la-normalizzazione-per-eta-accademica-secondo-anvur-ovvero-summum-jus-summa-iniuria/ (ultimo accesso 9 settembre 2016).

${ }^{58}$ Per gli associati due monografie su temi diversi o una monografia di particolare valore, più almeno quattro articoli su altri temi, di cui minimo due in organi con peer review. Per gli ordinari accanto a tre monografie un'edizione (in Italia di norma curatele, ma nessuna edizione critica) o un gran numero di articoli su altri temi, di cui anche minimo due in organi con peer review. Alla prossima commissione resta la libertà ovviamente di cambiare tali criteri, e del resto la tendenza ad avere la mano libera con i criteri è prassi abituale anche delle commissioni tedesche.

${ }^{59}$ Patrizia Guarnieri, una storica decisamente valida, classe 1954, ha pubblicato fra il 1978 e il 2013 non meno di 14 libri e 145 articoli. In questo modo assume una posizione avvantaggiata fra i candidati, pur non costituendo in nessun modo un'eccezione. Di più all'indirizzo http:// www.unifi.it/p-doc2-2013-200049-G-3f2a3d323a2d2d.html (ultimo accesso 23 settembre 2016). 
sentare delle monografie a una casa editrice già prima di questa età non è ra$\mathrm{ro}^{60}$. Inoltre i libri voluminosi valgono di più dei libri sottili.

L'elevata produttività ha molte cause. Come già evidenziato, discorrere e scrivere fanno entrambi parte della tradizione culturale italiana. A questa appartiene molto spesso anche il riflettersi della gioia di raccontare in saggi o libri. Le opere impostate analiticamente sono decisamente rare, così come le considerazioni teoriche, finanche lo stato della ricerca viene indicato solo in casi eccezionali. I libri dispongono raramente di bibliografie, il che rende ancor più difficile la verifica del materiale citato; anche riportare dettagliatamente le fonti originali non costituisce la norma. Al contrario raramente manca un indice dei nomi, grazie al quale amici, aiutanti e santi protettori possono sapere con uno sguardo quante volte sono stati citati. Solo la metà dei candidati ha invece citato articoli in riviste con peer review anonima ${ }^{61}$. A monte ci sarebbe il fatto che a molti tali processi oggettivanti non piacciono e non promettono neppure alcun vantaggio nel quotidiano. Molte riviste sono ancora, come da prassi nel XIX secolo, punti d'incontro di amici e confratelli e perciò ci si considera, stando a quanto riportato occasionalmente in alcuni curricula presentati, collaboratori fissi di un periodico, sul quale si pubblica in maniera regolare. Non pochi di questi periodici si collocano al di fuori del mondo scientifico, ma chiaramente non al di fuori di quello accademico (come dimostra uno sguardo alla sempre ampia lista dei curatori), per cui gli articoli ivi pubblicati vengono ovviamente menzionati accanto a quelli su "Passato e presente" o in "Studi storici", due delle riviste principali.

Qui si riconosce un ordine di valori nettamente diverso da quello tedesco. Non è un ordine circoscritto all'università e al mondo scientifico, ma è un qualcosa che riflette emblematicamente le condizioni di vita italiane. In uno Stato, che nonostante tutta la modernizzazione dopo 150 anni non è ancora riuscito a garantire ai suoi cittadini condizioni di vita, e soprattutto servizi pubblici, accettabili, le relazioni personali sono fondamentali per la sopravvivenza. L'anonimato è dannoso, la fiducia in procedure regolamentate troppo spesso semplicemente ingenua. Anche nella ricerca scientifica si allacciano il più presto possibile relazioni stabili, il confine fra contatti professionali e clientelismo resta spesso poco chiaro. Ciò si evince da molti aspetti. Quasi ogni libro ha una premessa o un'introduzione, o entrambe, scritte da terzi; tesi di dottorato senza prefazione del relatore sono una grande eccezione e fanno presumere dei conflitti. In questi testi si riflette una parte di quelle reti di relazioni in cui vive l'autore.

${ }^{60}$ Giovanni Sbordone, classe 1972, dottore di ricerca dal 2010, a quella data aveva già pubblicato quattro libri, per non parlare degli articoli. Su di lui in Wikipedia, http://it.wikipedia.org/ wiki/Giovanni_Sbordone (ultimo accesso 9 settembre 2016).

${ }^{61} \mathrm{Nel}$ campione preso ad esempio sono 23 su 54 aspiranti a un posto da professore associato e 8 su 15 aspiranti a un posto da ordinario. Vi sono ad ogni modo ben poche riviste storiche che permettono la valutazione esterna. Un rilevamento del 2003 ne faceva emergere appena due. Cfr. Cavazza, Die Neueste Geschichte, cit., p. 108, nota 74. 
Cose di questo genere si trovano naturalmente anche altrove. Tipico dell'Italia è però non solo la frequenza di questi fattori, ma anche il fatto che quasi ogni libro appare in una collana, il che porta allo scoperto ulteriori reti di rapporti, volti a rendere possibile l'accesso a finanziamenti per i costi di pubblicazione. Questo perché molto raramente entrano in gioco il Miur o il $\mathrm{Cnr}^{62}$. Più di questi partecipano ai finanziamenti i dipartimenti, gli istituti, le organizzazioni per le pari opportunità, enti di ricerca, fondazioni, sindacati, associazioni, cooperative e banche (obbligate dalla legge), soprattutto però comuni, province e regioni e questo si traduce una volta di più nell'imprescindibilità dei contatti presso tali finanziatori. A ogni modo condizioni del genere richiedono inevitabilmente già nel quotidiano una forte tendenza al localismo. A tal proposito a occhio e croce il 10 per cento dei candidati fa intravedere sul piano biografico, professionale e scientifico un perfetto esempio di localismo. Lidea che da ciò ne possa soffrire la qualità gli è estranea o comunque irrilevante, dal momento che le strette interconnessioni sul piano locale garantiscono la sopravvivenza più di quanto faccia un confronto a livello nazionale.

Il numero dei premi in Italia è di gran lunga superiore a quello della Germania, cosicché circa la metà di tutti i candidati ha ricevuto almeno un premio. Non tutti i premi portano denaro in dote, ma alcuni ne forniscono comunque abbastanza da permettere di finanziare la stampa di un libro. Vi sono perciò case editrici che fondano sedi distaccate in quelle località che impongono l'investimento in loco dei ricchi premi messi in palio $^{63}$. In non pochi premi il rapporto fra tema e onorificenza è palese, tanto da rendere scontata la domanda se un'opera premiata in tal modo davvero sia nata in maniera non premeditata e semplicemente orientata all'oggetto della ricerca. In ogni caso chi lavori su Nilde Iotti, la collaboratrice e compagna di vita di Palmiro Togliatti, a lungo segretario generale del Pci, ottiene quasi immancabilmente il Premio Nilde Iotti per la storia contemporanea, chi scrive dei fratelli Rosselli riceve con grande probabilità il premio alla loro memoria messo in palio dalla Fondazione fratelli Rosselli di Firenze.

Una gran parte dei premi favorisce il localismo ${ }^{64}$, anche in quei casi in cui vengono spacciati per "nazionali". Un ricercatore proveniente da Senigallia,

${ }^{62} \mathrm{Il} \mathrm{Cnr} \mathrm{come} \mathrm{ente} \mathrm{assomiglia,} \mathrm{con} \mathrm{i} \mathrm{suoi} \mathrm{progetti} \mathrm{di} \mathrm{ricerca,} \mathrm{alla} \mathrm{Max} \mathrm{Planck-Gesellschaft}$ e come questa è solo da relativamente poco tempo impegnato nelle scienze umane. Tuttavia dal punto di vista del livello di organizzazione, dell'autorappresentazione e del prestigio internazionale intercorre fra i due una differenza enorme.

${ }^{63}$ Per esempio la Donzelli di Roma. La casa editrice ha fondato la Meridiana Libri a Catanzaro, poiché il remunerativo Premio letterario città di Catanzaro esige che la somma del premio sia utilizzata in loco.

${ }^{64}$ In linea di massima la situazione è simile anche nella storia regionale tedesca; anche lì è da notare l'assegnazione di molti premi, ovviamente solo a lavori provenienti dalle proprie schiere. Tuttavia, come già sottolineato, la storia regionale del XIX e soprattutto del XX secolo non gode in Germania di una visibilità neppure lontanamente paragonabile a quella che ha in Italia. 
in provincia di Ancona, che ha compiuto studi quasi esclusivamente sulle élite politiche delle Marche, ha ottenuto nel 1999 il Premio nazionale di cultura "Frontino Montefeltro", a proposito del quale la minuscola comunità montana di Frontino, situata nell'entroterra marchigiano, dichiara fiera su internet: "Il Premio nazionale di cultura Frontino Montefeltro ha superato da tempo i confini dell'antico ducato dei Federico da Montefeltro e quelli della regione, per assurgere a pieno merito come uno degli appuntamenti culturali di rilevanza nazionale per merito anche dell'illustre giuria, presieduta dal magnifico rettore dell'Università degli studi di Urbino Carlo Bo"65. Invece una storica sociale proveniente da Pisa e impiegata in quell'università come ricercatrice dal 1980, per la raccolta da lei curata sulla "fabbrica di Boccadarno", che si trovava un tempo a Pisa, ha ricevuto nel 2009 il Premio nazionale di narrativa e saggistica "Il delfino", gestito e assegnato ogni anno dalla pro loco del litorale pisano nell'ambito del suo programma estivo.

Ovviamente non tutte le storie regionali favoriscono il localismo, ma il grado di autoreferenzialità in questo settore è tradizionalmente alto. Nell'ottica della crisi ormai pluridecennale dello Stato centrale italiano la tendenza a servirsi del localismo è piuttosto evidente, tanto da poter parlare di un boom della ricerca localistica, segnatamente nel Mezzogiorno. L'autoreferenzialità può essere osservata però anche in altri ambiti. Questa è stata per lungo tempo caratteristica della storia del movimento operaio, dei sindacati o dei partiti di sinistra, in Italia come in Germania, sebbene anche in Italia nel frattempo corra il rischio di scomparire. Le organizzazioni cristiano-democratiche hanno superato invece abbastanza bene il crollo della Democrazia cristiana, tant'è che circa due dozzine di candidati si dedicano in maniera più o meno completa alla loro storia e a tal fine vengono da queste sostenuti. Anche nel caso dei lavori sul cattolicesimo la gamma di qualità è notevole. Accanto a buone ricerche sulla storia della chiesa, degli ordini religiosi, delle missioni e delle religioni in Italia e all'estero, ve ne sono non poche affatto problematiche prodotte da storici al servizio della chiesa, i quali in maniera palese rifiutano le problematiche della ricerca moderna e anzi, in sintesi, producono da devoti per i devoti. A Foggia, presso l'Istituto superiore di scienze religiose ${ }^{66}$, è in servizio un docente pugliese di Storia della chiesa, munito di due dottorati di ricerca (presso la Cattolica di Milano e la Gregoriana di Roma, due rinomate università cattoliche), dove compie ricerche prevalentemente sulla chiesa e sul personale ecclesiastico in Puglia, pubblicando anche nelle riviste locali "Quis ut Deus" o "Studi su padre Pio". Non è certo una cosa deprecabile. Tuttavia presentare seriamente qualcosa del genere come esempi di un'eccellenza scientifica lascia intravedere un ordine di valori ben lontano dagli standard consueti.

${ }^{65} \mathrm{Si}$ veda http://www.frontinomontefeltro.it/eventi/premio-nazionale-di-cultura-2011 (ultimo accesso 11 settembre 2016). Vincitore del premio fu Marco Severini.

${ }^{66}$ Chiunque, anche un professore, sia impiegato presso un'università cattolica o privata e intenda presentare domanda presso un'università pubblica, deve attestare il titolo di abilitazione. 
Un'immagine simile è fornita da un candidato, anch'egli dotato di un dottorato di ricerca presso la Cattolica, a dire il vero con una ricerca seria, il quale attualmente si occupa a Brescia del giovane Montini, il futuro papa Paolo VI, e in pratica ha pubblicato tutti i suoi 29 articoli, sempre di poche pagine, sui quaderni locali bresciani "C\&D: Città e dintorni e Notiziario - Istituto Paolo VI", di cui è anche condirettore. Inoltre cura i discorsi e gli scritti di Montini come arcivescovo di Milano, la sua corrispondenza con Maritain e una delle sue encicliche. Qualora vi fossero anche in Italia facoltà teologiche con cattedre di Storia della chiesa, questo candidato non sarebbe costretto a collocarsi fra gli storici contemporanei. La scarsa differenziazione organizzativa della storiografia italiana fa sì che esperti di storia locale o storici della chiesa, che seguono altre logiche e servono un altro pubblico, vengano ascritti a essa. Non sempre ciò migliora il prestigio di questa disciplina.

\section{Internazionalizzazione}

Resta ancora la questione che sta più a cuore a un osservatore straniero, se i ricercatori italiani guardino al di là dei propri confini nazionali e, in caso affermativo, in quale direzione. Non andrebbe dimenticato che la ricerca del passato e in particolare la sua narrazione serve da sempre alla comprensione di sé sul piano nazionale e che solo il paese d'immigrazione per eccellenza, gli Stati uniti, si concede il "lusso" di disporre di storici specializzati in pratica per ogni Paese del pianeta. La norma è rappresentata dall'occuparsi della storia del proprio paese ed è difficile sviluppare un criterio che fissi un limite accettabile al narcisismo nazionale. È perciò sorprendente che nel campione degli aspiranti al posto di lavoro di professore ordinario solo in tre forniscano contributi alla storia extraitaliana e anche con una visione di largo respiro: l'unificazione europea, l'Etiopia come oggetto della storia delle missioni e Israele. Un'interpretazione razionale di questa circostanza non è possibile, dal momento che si tratta apertamente di risultati fortuiti — poiché fra tutti i candidati si trovano lavori di rinomati ricercatori sulla storia soprattutto dei paesi europei confinanti ${ }^{67}$ - , ma non può trattarsi solo di una coincidenza. Dipende dall'età? La media per il concorso del 2012 è di cinquant'anni e per quello del 2013 ancora di 48 anni. Ciò mostra sia l'ingorgo negli avanzamenti di carriera sia l'appartenenza all'ultima generazione precedente all'epoca globalizzata. Confrontarsi con altri Paesi e culture non comporta evidentemente alcun vantaggio. In realtà i curricula delle scuole di dottorato contengono spesso le parole chiave "Europa" o "Mediterraneo"68 — c'è da chiedersi automaticamente chi in quelle sedi

${ }^{67}$ Da citare sono qui solo alcuni nomi, ben noti anche in Germania grazie a pubblicazioni tedesche. Si tratta di Stefano Cavazza, Monica Cioli, Costanza D’Elia e Filippo Focardi.

${ }^{68}$ Cfr. nota 38 . 
offra corsi corrispondenti - cosicché si sarebbe portati a pensare di poter collocare le persone formate in tali scuole nell'ambito della storia di altre nazioni. Più verosimilmente guardando ai casi in cui qualcuno nel campione preso in esame abbia scelto un tema di storia extraitaliana pare abbiano pesato ragioni personali o motivazioni non desumibili, quindi casuali. Nel 2013 l'orizzonte si allarga ed emergono finalmente anche contributi su altri paesi e non solo storie di relazioni. Vi sono contributi sull'Unione sovietica, sul tardo Impero ottomano, sull'Asia centrale e in particolare sul Pakistan, naturalmente anche sempre sull'Europa, sulla quinta repubblica francese e quattro persone contemporaneamente si occupano della Germania. Anche in questi casi una spiegazione non è facile. La Francia soprattutto riceve scarsa attenzione, se si osserva quanti candidati abbiano ottenuto la Maîtrise o il D.e.a. o addirittura abbiano sostenuto l'esame per il Maître de conférences, mentre tre dei quattro candidati che si occupano della storia tedesca hanno anche conseguito il dottorato in Germania. Solo una cosa è chiara: il campione del 2013 è in media decisamente più giovane (38 anni: nel 2212 la media era ancora di 45 anni). Che qui vi sia un rapporto causale è avvallato da molti fattori.

\section{Fosche prospettive?}

Cosa dire quindi per un bilancio generale? Cosa si evince sulla ricerca italiana nella storia contemporanea dal campione qui offerto, che parla di coloro che attualmente aspirano a un posto di lavoro da professore? Tra questi si trova una schiera di ottime ricercatrici e ottimi ricercatori, che recano giovamento e fanno onore alle università italiane. Citarli per nome è impossibile per diversi motivi, quindi si indicheranno i criteri: pubblicazioni in riviste italiane ed estere verificate mediante peer review, inviti presso istituzioni scientifiche straniere e collaborazioni con quegli istituti di ricerca italiani veramente degni di tale nome. La maggior parte di loro, non c'è da sorprendersi, è di casa in università del centro e nord Italia: Bologna, Torino, Milano, Pisa, Firenze - in quest'ordine; anche da Roma e Napoli provengono alcuni candidati eccellenti. La massa degli storici contemporaneisti invece non è per nulla innovativa nei contenuti e metodologicamente e opera prevalentemente in senso narcisistico nell'ambito della storia politica del dopoguerra ${ }^{69}$. Neppure una volta la "scoperta" della nazione portata avanti da Alberto Maria Banti per il Risorgimento è stata utilizzata nell'ambito della storia repubblicana, sebbene ciò rappresenterebbe una via per confutare la tesi mediatica proposta da De Felice e Galli della Loggia sulla morte della nazione risalente all' 8 settembre $1943^{70}$. La storia delle idee si rin-

\footnotetext{
${ }^{69}$ Cavazza giunge a un giudizio simile, ma intravede netti margini di miglioramento; cfr. Id., Die Neueste Geschichte, cit., p. 105 sg.

${ }^{70} \mathrm{Si}$ veda Renzo De Felice, Rosso e Nero, a cura di Pasquale Chessa, Milano, Baldini \& Castoldi, 1995; Ernesto Galli della Loggia, L'identità italiana, Bologna, il Mulino, 1998.
} 
nova a malapena a causa del persistente strapotere della tradizione di Benedetto Croce e Antonio Gramsci. La moderna storia sociale non ha avuto una marcia trionfale simile a quella della controparte tedesca, perché non garantita da potenti interpretazioni come quella del Sonderweg. Una storia delle migrazioni estesa a livello globale, con uno sguardo alla diaspora del commercio e dei suoi prodotti o ai gruppi o minoranze transnazionali è ostacolata dalla salda posizione della storia classica dell'emigrazione e le parole chiave dell'antropologia storica — alterità, ibridazione, quotidianità — si incontrano decisamente poco nei lavori di storia contemporanea. Lo slancio verso nuovi orizzonti non appare essere al momento un segno distintivo della storiografia contemporanea italiana, quantomeno di questo campione di candidati. La cosa non dipende soltanto dalla carenza di fondi e finanziamenti; Israele, che è altrettanto parsimoniosa nel finanziare le sue università e le facoltà di lettere in particolare, si trova in una posizione ben diversa. Il tracollo è dato dalla crisi della cultura accademica fin dagli anni Settanta, parallela a quella della politica. Cooptazioni e chiamate a livello locale sono la morte dell'università, a meno che le disposizioni non siano estremamente rigorose, come in Inghilterra e negli Stati uniti. Non è certo il caso dell'Italia, dove al contrario, secondo il parere degli stessi osservatori italiani, il familismo e il clientelismo nel frattempo minacciano di sfaldare anche il sistema dell'istruzione ${ }^{71}$. Nel Paese delle "cento città" e di un cattolicesimo oggi come in passato ancora fortemente radicato culturalmente, la tentazione di condurre la storia sul piano di un'autorappresentazione locale o religiosa è grande. Per altri si tratta solo di dare sfogo a un interesse antiquario. Mancano del tutto numerose sottodiscipline, a volte anche a causa della scarsa conoscenza della lingua inglese. C'è da stupirsi se quasi i due terzi dei candidati, persone quindi che spesso hanno già iniziato una carriera universitaria o che comunque aspirano a essa, secondo il parere della commissione non abbiamo esaudito i criteri stabiliti ${ }^{72}$ ?

71 "Familismo e clientelismo partitico sono tumori che ammorbano l'intero mercato del lavoro, ma proprio nel sistema della formazione essi hanno trovato negli ultimi decenni un'applicazione sistematica"; Mondini, Qualità vo cercando, cit., p. 1. Un altro osservatore si esprime in maniera ancora più netta: "Di chi è la colpa? Forse, anche di quel sistema di cooptazione clientelare e feudale che ha trasformato molti dipartimenti universitari in uffici di collocamento per portaborse"; Novello Monelli, Una modesta proposta. Se scuola e università sono campo di battaglia per estremismi opposti, "Mente politica", 25 settembre 2014, http://www.mentepolitica.it/articolo/una-modesta-proposta/208 (ultimo accesso 12 settembre 2016). In altro luogo, sempre di "Mente politica", scrive Monelli, Come ti rottamo l'università, p. 2: "un perverso meccanismo di reclutamento localistico ha trasformato molte facoltà soprattutto dagli anni Novanta in avanti nella culla del clientelismo e del familismo amorale"; "familismo amorale" è un termine coniato dall'antropologo americano E.C. Banfield nel 1958, al fine di descrivere l'assenza di orientamento al senso comune della società dell'Italia meridionale, con la conseguenza di un permanente ritardo; da allora è divenuto un termine proverbiale, anche se nel frattempo quest'analisi va discussa in modo critico.

${ }^{72}$ Questa condizione trova senza sorpresa una corrispondenza nel carente livello di studi della gran parte dei diplomati italiani. La ricerca sull'istruzione ha stabilito che in Italia già nei tar- 
Allora che si fa? Chi controlla che i bocciati vengano tenuti lontani dall'occupazione di posti? Il ministero competente no di certo, dato che non si è nemmeno arrischiato a escludere dai concorsi coloro che da lui stesso fossero giudicati incapaci di soddisfare i requisiti quantitativi minimi, bensì ha passato ogni candidato alla commissione seguendo il principio diffuso del todos caballeros. Da sinistra alcuni critici conducono nel nome della lotta contro il neoliberalismo una campagna dai toni manichei contro i controlli di qualità e il "lavoro a cottimo" 73 e cercano in tal modo di impedire l'affermarsi di standard adeguati alla ricerca scientifica e riconosciuti internazionalmente. Ricercatori universitari italiani rigorosi e capaci nei colloqui diretti si sono mostrati vicini alla disperazione, non hanno partecipato in maniera seria a questo procedimento e continuano a cercare una sistemazione all'estero ${ }^{74}$. Questo articolo purtroppo non può fornire loro grandi speranze.

(traduzione di Pierluigi Pironti)

di anni Settanta le richieste scolastiche al momento dell'esame di maturità sono state spostate al ribasso, al fine di raggiungere le quote di diplomati stabilite dagli standard (erronei) dell'Osce. "Il fatto che oggi due terzi di un'annata ottenga un titolo di studio è in totale contrasto con i risultati PISA, secondo i quali solo un quarto degli studenti è da considerarsi efficiente"; si veda Rainer Bölling, Viele Abiturienten, weniger Bildung, "Frankfurter Allgemeine Zeitung", 4 dicembre 2014.

${ }^{73} \mathrm{Si}$ veda Valeria Pinto, Valutare e punire. Una critica della cultura della valutazione, Napoli, Cronopio, 2012; Giulio Palermo, Baroni e portaborse. I rapporti di potere nell'università, Roma, Editori internazionali riuniti, 2012.

${ }^{74}$ Emblematica la risposta di Elena Mazzini, abilitatasi poi con successo, alla domanda se, nel caso di una seconda opportunità, avrebbe intrapreso nuovamente la strada del dottorato e della carriera accademica: "Si [...]. A ogni modo cercherei di legare la mia attività di ricerca all'estero, non perché credo al motto che all'estero sia tutto migliore, bensì perché il mercato del lavoro in Italia è saturo e la formazione postlaurea non è riconosciuta come tale. In essa si intravede invece una qualificazione secondaria a stento meritata, soprattutto quando la si è conseguita in una disciplina umanistica". Si veda Zur Lage der Nachwuchsforschung, cit., p. 25. 